\title{
ON MAXIMAL GREEN SEQUENCES
}

\author{
T. BRÜSTLE, G. DUPONT AND M. PÉROTIN
}

\begin{abstract}
Maximal green sequences are particular sequences of quiver mutations appearing in the context of quantum dilogarithm identities and supersymmetric gauge theory.

Interpreting maximal green sequences as paths in various natural posets arising in representation theory, we prove the finiteness of the number of maximal green sequences for cluster finite quivers, affine quivers and acyclic quivers with at most three vertices. We also give results concerning the possible numbers and lengths of these maximal green sequences.
\end{abstract}

\section{INTRODUCTION}

A maximal green sequence is a certain sequence of quiver mutations given by a sequence $\mathbf{i}=\left(i_{1}, \ldots, i_{l}\right)$ of vertices in a quiver $Q$. The term "maximal green sequence" has been coined by Keller in [26] where these sequences are used to obtain quantum dilogarithm identities and refined Donaldson-Thomas invariants. In fact, each sequence $\mathbf{i}$ defines an element $\mathbb{E}(\mathbf{i})$ in the formal quantum affine space $\mathbb{A}_{Q}$ of $q$-commuting variables, and it has been shown in [26] that $\mathbb{E}(\mathbf{i})=\mathbb{E}\left(\mathbf{i}^{\prime}\right)$ whenever $\mathbf{i}, \mathbf{i}^{\prime}$ are maximal green sequences of a Dynkin quiver. For the quiver $Q$ with two vertices and one arrow between them (i.e. $Q$ is of type $A_{2}$ ), there are exactly two maximal green sequences $\mathbf{i}$ and $\mathbf{i}^{\prime}$ of respective lengths two and three, and in this case the equality $\mathbb{E}(\mathbf{i})=\mathbb{E}\left(\mathbf{i}^{\prime}\right)$ is a quantum analog of the pentagon identity for the Rogers dilogarithm. Even in the non-Dynkin case, the invariants $\mathbb{E}(\mathbf{i})$ can be interpreted as refined DT-invariants following work of Kontsevich-Soibelman; we refer to [26] for a thorough explanation of these ideas.

Independently, the same sequences of quiver mutations are studied in theoretical physics where they yield the complete spectrum of BPS states, see [3, 11].

Maximal green sequences can also be interpreted as maximal chains in a partially ordered set that arises from a cluster exchange graph once an initial seed is fixed. This partial order relation has earlier been studied by Happel and Unger on a subgraph of the cluster exchange graph [47, 48, 23, and recently a number of representation-theoretic interpretations of the poset structure of the whole cluster exchange graph has been given [34, 31, 32, 11.

The theory of cluster algebras is related to numerous other fields, and thus the cluster exchange graph can be interpreted in many ways. For instance one can view it as a generalised associahedron, which is known to carry a poset structure (the Tamari poset). However, we will focus in this paper mainly on the combinatorial description of the poset structure by quiver mutations as given in [26, and we intend to initiate a systematic study of maximal green sequences applying representation-theoretic techniques.

Organisation of the article. In Section 2 we introduce the notion of maximal green sequences in elementary terms and present some general results. When the proofs do not require any further background we present them in this section. When they do require some additional background, they are postponed to Section 8

The short Section 3 makes the appearance of maximal green sequences explicit in the context of theoretical physics.

Date: March 1, 2013.

2010 Mathematics Subject Classification. Primary: 13F60. Secondary: 16G20, 81T60. 
In Section 4, we study maximal green sequences for quivers of finite cluster type. As before, the proofs requiring additional background are postponed to Section 9 .

Section 5 presents an analysis of the maximal green sequences for acyclic quivers of infinite representation types; the corresponding proofs are found in Section 10.

The representation-theoretical background underlying the proofs and (part of) the motivations of this article can be found in Section 6 where we recall the various connections between maximal green sequences and some classical posets in representation theory.

In this spirit, we present in Section 7 additional results on the connections between maximal green sequences and the classical Happel-Unger poset of tilting modules over an algebra, see [23].

Sections 810 contain the missing proofs.

Finally, in Appendix A we present certain explicit examples which were computed with the Quiver Mutation Explorer, see [16.

\section{Green SEQUEnCES}

Without further specification, quivers will always be finite connected oriented graphs and cluster quivers will be quivers without loops or oriented 2-cycles. A quiver is called acyclic if it has no oriented cycles. Given a quiver $Q$, we denote by $Q_{0}$ its set of vertices and by $Q_{1}$ its set of arrows.

2.1. Cluster algebras. Introduced in [18, cluster algebras are commutative rings equipped with a distinguished set of generators, the cluster variables, gathered into possibly overlapping subsets of pairwise compatible variables, the clusters, defined recursively by a combinatorial process, the mutation. The dynamics of this mutation process are encoded in a combinatorial datum, the exchange matrix.

An exchange matrix is a matrix $B=\left(b_{i j}\right) \in M_{n, n+m}(\mathbb{Z})$ for some $m, n \geqslant 0$ such that the principal part of $B$, that is, the square submatrix $B^{0}=\left(b_{i j}\right)_{1 \leqslant i, j \leqslant n} \in M_{n}(\mathbb{Z})$ is skew-symmetrisable, that is, there exists a diagonal matrix $D \in M_{n}(\mathbb{Z})$ with positive diagonal entries such that $D B^{0}$ is skew-symmetric. Abusing terminology we say that $B$ itself is skew-symmetrisable, or that it is skew-symmetric when $B^{0}$ is so.

Given a skew-symmetrisable exchange matrix $B \in M_{n, n+m}(\mathbb{Z})$, we denote by $\mathcal{A}_{B}$ the corresponding cluster algebra, see 20] for details.

Definition 2.1 (Matrix mutation). Let $B \in M_{n, n+m}(\mathbb{Z})$ be skew-symmetrisable. Then for any $1 \leqslant k \leqslant n$, the mutation of $B$ in the direction $k$ is the skew-symmetrisable matrix $\mu_{k}(B)=\left(b_{i j}^{\prime}\right) \in M_{n, n+m}(\mathbb{Z})$ given by

$$
b_{i j}^{\prime}= \begin{cases}-b_{i j} & \text { if } i=k \text { or } j=k \\ b_{i j}+\left[b_{i k}\right]_{+}\left[b_{k j}\right]_{+}-\left[b_{i k}\right]_{-}\left[b_{k j}\right]_{-} & \text {otherwise }\end{cases}
$$

where $[x]_{+}=\max (x, 0)$ and $[x]_{-}=\min (x, 0)$ for any $x \in \mathbb{Z}$.

It is easy to see that $\mu_{k}\left(\mu_{k}(B)\right)=B$ for any $1 \leqslant k \leqslant n$ and that $\mu_{k}(B)$ is skew-symmetric if and only if $B$ is skew-symmetric. In this latter case, we say that $\mathcal{A}_{B}$ is simply-laced and it is usually more convenient to use the formalism of ice quivers instead of exchange matrices.

2.2. Ice quivers and their mutations. An ice quiver is a pair $(Q, F)$ where $Q$ is a cluster quiver and $F \subset Q_{0}$ is a (possibly empty) subset of vertices called the frozen vertices such that there are no arrows between them. For simplicity, we always assume that $Q_{0}=\{1, \ldots, n+m\}$ and that $F=$ $\{n+1, \ldots, n+m\}$ for some integers $m, n \geqslant 0$. If $F$ is empty, we simply write $Q$ for $(Q, \varnothing)$.

We associate to $(Q, F)$ its adjacency matrix $B(Q, F)=\left(b_{i j}\right) \in M_{n, n+m}(\mathbb{Z})$ such that

$$
b_{i j}=\left|\left\{i \longrightarrow j \in Q_{1}\right\}\right|-\left|\left\{j \longrightarrow i \in Q_{1}\right\}\right|
$$

for any $1 \leqslant i \leqslant n$ and any $1 \leqslant j \leqslant n+m$.

The map $(Q, F) \mapsto B(Q, F)$ induces a bijection from the set of ice quivers to the set of skew-symmetric exchange matrices. Therefore, to any ice quiver $(Q, F)$ we can associate the cluster algebra $\mathcal{A}_{(Q, F)}=$ $\mathcal{A}_{B(Q, F)}$. 
Definition 2.2 (Quiver mutation). Let $(Q, F)$ be an ice quiver and $k \in Q_{0}$ be a non-frozen vertex. The mutation of $Q$ at $k$ is defined as the ice quiver $\left(\mu_{k}(Q), F\right)$ where $\mu_{k}(Q)$ is obtained from $Q$ by applying the following modifications:

(1) For any pair of arrows $i \stackrel{a}{\rightarrow} k \stackrel{b}{\rightarrow} j$ in $Q$, add an arrow $i \stackrel{[a b]}{\longrightarrow} j$ in $\mu_{k}(Q)$;

(2) Any arrow $i \stackrel{a}{\rightarrow} k$ in $Q$ is replaced by an arrow $i \stackrel{a^{*}}{\longleftarrow} k$ in $\mu_{k}(Q)$;

(3) Any arrow $k \stackrel{b}{\rightarrow} j$ in $Q$ is replaced by an arrow $k \stackrel{b^{*}}{\longleftarrow} j$ in $\mu_{k}(Q)$;

(4) A maximal collection of 2-cycles is removed as well as all the arrows between frozen vertices.

Then it is easy to see that for any non-frozen vertex $k \in Q_{0}$, the ice quiver $\mu_{k}(Q, F)$ is the ice quiver corresponding to the skew-symmetric matrix $\mu_{k}(B(Q, F))$.

Example 2.3. Figure 1 shows an example of successive quiver mutations.
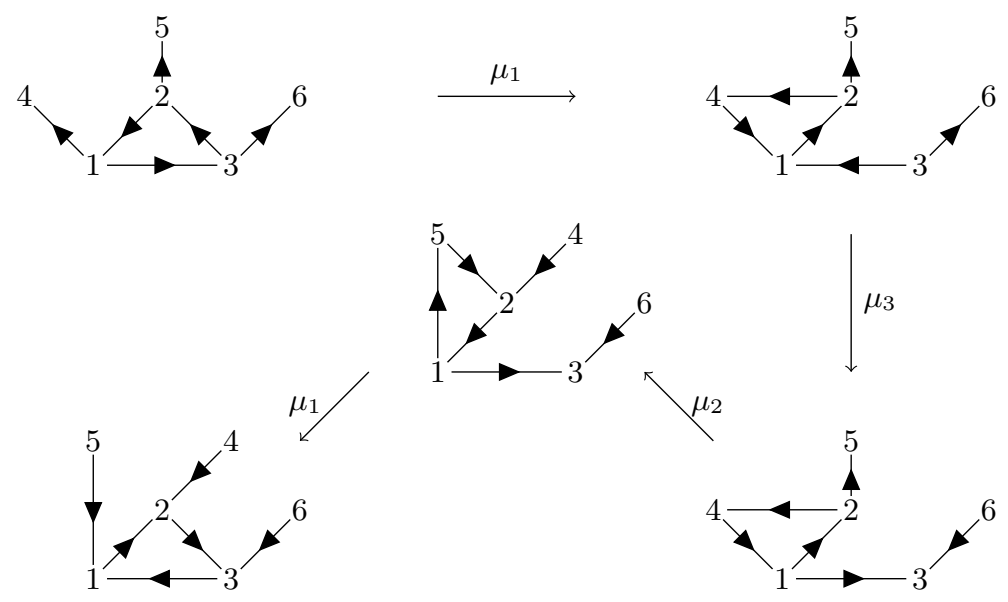

FIGURE 1. An example of quiver mutations.

Two ice quivers are called mutation-equivalent if one can be obtained from the other by applying a finite number of successive mutations at non-frozen vertices. Since mutations are involutive, this defines an equivalence relation on the set of ice quivers. The equivalence class of an ice quiver $(Q, F)$ is called its mutation class and is denoted by $\operatorname{Mut}(Q, F)$.

Two ice quivers $(Q, F)$ and $\left(Q^{\prime}, F\right)$ sharing the same set of frozen vertices are called isomorphic as ice quivers if there is an isomorphism of quivers $\phi: Q \longrightarrow Q^{\prime}$ fixing $F$. In this case, we write $(Q, F) \simeq\left(Q^{\prime}, F\right)$ and we denote by $[(Q, F)]$ the isomorphism class of the ice quiver $(Q, F)$.

2.3. Green sequences. From now on, $Q$ will always denote a cluster quiver and we fix a copy $Q_{0}^{\prime}=$ $\left\{i^{\prime} \mid i \in Q_{0}\right\}$ of the set $Q_{0}$ of vertices in $Q$. We will identify $Q_{0}$ with the set of integers $\{1, \ldots, n\}$ and $Q_{0}^{\prime}$ with $\{n+1, \ldots, 2 n\}$ in such a way that for any $1 \leqslant i \leqslant n$, we have $i^{\prime}=n+i$.

Definition 2.4 (Framed and coframed quivers). The framed quiver associated with $Q$ is the quiver $\widehat{Q}$ such that:

$$
\begin{aligned}
& \widehat{Q}_{0}=Q_{0} \sqcup\left\{i^{\prime} \mid i \in Q_{0}\right\}, \\
& \widehat{Q}_{1}=Q_{1} \sqcup\left\{i \longrightarrow i^{\prime} \mid i \in Q_{0}\right\} .
\end{aligned}
$$


The coframed quiver associated with $Q$ is the quiver $\breve{Q}$ such that:

$$
\begin{aligned}
& \check{Q}_{0}=Q_{0} \sqcup\left\{i^{\prime} \mid i \in Q_{0}\right\}, \\
& \check{Q}_{1}=Q_{1} \sqcup\left\{i^{\prime} \longrightarrow i \mid i \in Q_{0}\right\} .
\end{aligned}
$$

If $Q$ is an arbitrary cluster quiver, both $\widehat{Q}$ and $\breve{Q}$ are naturally ice quivers with frozen vertices $Q_{0}^{\prime}$. Therefore, by $\operatorname{Mut}(\widehat{Q})$ we always mean the mutation class of the ice quiver $\left(\widehat{Q}, Q_{0}^{\prime}\right)$.

Definition 2.5 (Green and red vertices). Let $R \in \operatorname{Mut}(\widehat{Q})$. A non-frozen vertex $i \in R_{0}$ is called green if

$$
\left\{j^{\prime} \in Q_{0}^{\prime} \mid \exists j^{\prime} \longrightarrow i \in R_{1}\right\}=\varnothing \text {. }
$$

It is called red if

$$
\left\{j^{\prime} \in Q_{0}^{\prime} \mid \exists i \longrightarrow j^{\prime} \in R_{1}\right\}=\varnothing .
$$

If $R$ is an ice quiver in $\operatorname{Mut}(\hat{Q})$ with adjacency matrix $B=\left(b_{i j}\right) \in M_{n, 2 n}(\mathbb{Z})$, the submatrix $\mathbf{c}(R)=$ $\left(b_{i, n+j}\right)_{1 \leqslant i, j \leqslant n}$ is called the c-matrix of $R$. For any non-frozen vertex $i \in Q_{0}$, its $i$ th row $\mathbf{c}_{i}(R)$ is called the $i$ th $\mathbf{c}$-vector of $R$ and it encodes the number of arrows between $i$ and the frozen vertices in $R$. For instance, we have $\mathbf{c}(\hat{Q})=I_{n}$ and $\mathbf{c}(\breve{Q})=-I_{n}$. For more details on c-vectors, we refer the reader to [20] where they were introduced and to [37, 36, 46, 35, 27] where they were studied.

With this terminology, for a quiver $R \in \operatorname{Mut}(\widehat{Q})$, a vertex $i \in Q_{0}$ is green if and only if the $i$ th c-vector $\mathbf{c}_{i}(R)$ has only non-negative entries and it is red if and only if $\mathbf{c}_{i}(R)$ has only non-positive entries.

Given a quiver $R \in \operatorname{Mut}(\widehat{Q})$, we denote by $g(R)$ the number of green vertices in $R$. Note that this number only depends on $[R]$ so that we set $g([R])=g(R)$.

Theorem 2.6. Let $Q$ be a cluster quiver and $R \in \operatorname{Mut}(\widehat{Q})$. Then any non-frozen vertex in $R_{0}$ is either green or red.

Proof. Let $R \in \operatorname{Mut}(\widehat{Q})$. We need to prove that each row of the c-matrix of $R$ is non-zero and that its entries are either all non-negative or all non-positive. This result, known as the sign-coherence for c-vectors, was established in the case of skew-symmetric exchange matrices in [15].

For skew-symmetrisable exchange matrices the sign-coherence for c-vectors is still conjectural, and so is the non-simply-laced analogue of Theorem 2.6. For partial results concerning this, one might refer to [13.

Example 2.7. In $\widehat{Q}$, every non frozen vertex is green. In $\breve{Q}$, any non-frozen vertex is red.

Definition 2.8 (Green sequences, [26]). A green sequence for $Q$ is a sequence $\mathbf{i}=\left(i_{1}, \ldots, i_{l}\right) \subset Q_{0}$ such that $i_{1}$ is green in $\widehat{Q}$ and for any $2 \leqslant k \leqslant l$, the vertex $i_{k}$ is green in $\mu_{i_{k-1}} \circ \cdots \circ \mu_{i_{1}}(\widehat{Q})$. The integer $l$ is called the length of the sequence $\mathbf{i}$ and is denoted by $\ell(\mathbf{i})$.

A green sequence $\mathbf{i}=\left(i_{1}, \ldots, i_{l}\right)$ is called maximal if every non-frozen vertex in $\mu_{\mathbf{i}}(\widehat{Q})$ is red, where $\mu_{\mathbf{i}}(\widehat{Q})=\mu_{i_{l}} \circ \cdots \circ \mu_{i_{1}}(\widehat{Q})$

We denote by

$$
\text { green }(Q)=\left\{\mathbf{i}=\left(i_{1}, \ldots, i_{l}\right) \subset Q_{0} \mid \mathbf{i} \text { is a maximal green sequence for } Q\right\}
$$

the set of all maximal green sequences for $Q$.

Example 2.9. Figure 2 shows that the sequence of mutations considered in Figure 1 is a maximal green sequence for the oriented triangle. Frozen vertices are coloured in white, green vertices in green and red vertices in red.

We refer the reader willing to compute more examples to Bernhard Keller's java applet 24] or to the Quiver Mutation Explorer [16]. 

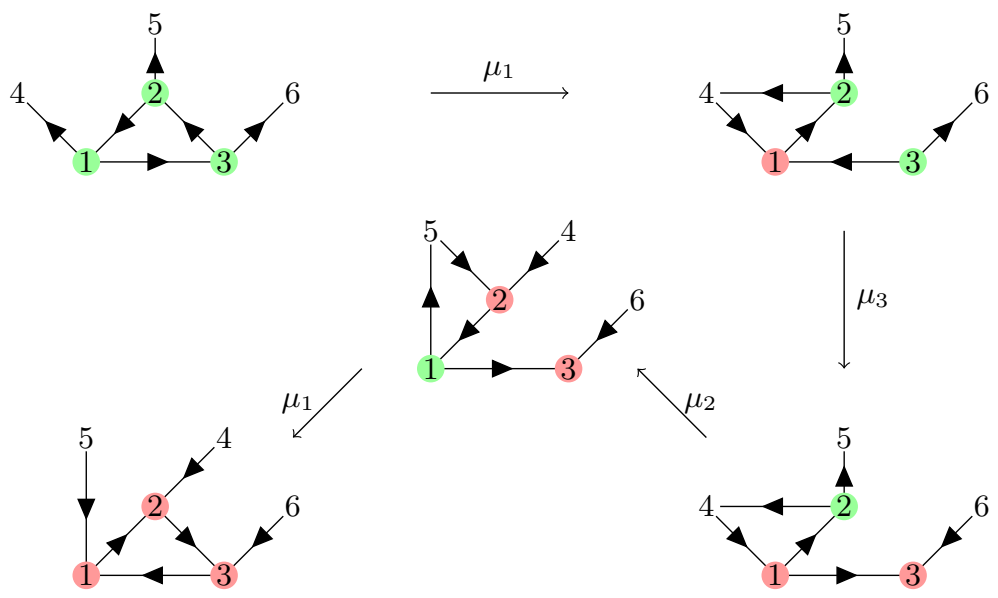

FiguRE 2. An example of a maximal green sequence.

2.4. The oriented exchange graph. The following proposition will be proved in Section 8 .

Proposition 2.10. Let $Q$ be a cluster quiver and let $R \in \operatorname{Mut}(\widehat{Q})$.

(1) If all the non-frozen vertices in $R_{0}$ are green, then $R \simeq \widehat{Q}$ as ice quivers.

(2) If all the non-frozen vertices in $R_{0}$ are red, then $R \simeq \check{Q}$ as ice quivers.

Definition 2.11 (Oriented exchange graph). The oriented exchange graph of $Q$ is the oriented graph $\overrightarrow{\mathbf{E G}}(Q)$ whose vertices are the isomorphism classes $[R]$ of ice quivers $R \in \operatorname{Mut}(\hat{Q})$ and where there is an arrow $[R] \longrightarrow\left[R^{\prime}\right]$ in $\overrightarrow{\mathbf{E G}}(Q)$ if and only if there exists a green vertex $k \in R_{0}$ such that $\mu_{k}(R) \simeq R^{\prime}$.

In [19], Fomin and Zelevinsky introduced the (unoriented) exchange graph of $Q$ as the dual graph $\mathbf{E G}(Q)$ of the cluster complex $\Delta\left(\mathcal{A}_{Q}\right)$ of the cluster algebra $\mathcal{A}_{Q}$ associated with $Q$. Vertices in $\mathbf{E G}(Q)$ are labelled by the clusters in $\mathcal{A}_{Q}$ and two clusters in $\mathbf{E G}(Q)$ are joined by an edge if and only if they differ by a single cluster variable. Then $\overrightarrow{\mathbf{E G}}(Q)$ is an orientation of $\mathbf{E G}(Q)$ corresponding to the choice of an initial seed in $\mathcal{A}_{Q}$ with exchange matrix $B(Q)$. The orientation is defined as follows. Let $\mathbf{x}$ and $\mathbf{x}^{\prime}$ be two adjacent clusters in $\mathbf{E G}(Q)$ corresponding respectively to $[R]$ and $\left[R^{\prime}\right]$ in $\overrightarrow{\mathbf{E G}}(Q)$. Assume that $\mathbf{x}$ and $\mathbf{x}^{\prime}$ differ by a single cluster variable $x_{i}$, so that $R^{\prime} \simeq \mu_{i}(R)$. Then the edge joining $\mathbf{x}$ and $\mathbf{x}^{\prime}$ in $\mathbf{E G}(Q)$ is oriented towards $\mathbf{x}^{\prime}$ if $i$ is green in $R$ and towards $\mathbf{x}$ otherwise.

As $\mathbf{E G}(Q)$ is an $n$-regular graph, if $[R]$ is a vertex in $\overrightarrow{\mathbf{E G}}(Q)$, then there are $g([R])$ arrows starting at $[R]$ in $\overrightarrow{\mathbf{E G}}(Q)$ and $n-g([R])$ arrows ending at $[R]$ in $\overrightarrow{\mathbf{E G}}(Q)$ (which, by Theorem 2.6, correspond to the red vertices in $R$ ).

Corollary 2.12. Let $Q$ be a cluster quiver. Then:

(1) $\overrightarrow{\mathbf{E G}}(Q)$ has a unique source, which is $[\hat{Q}]$.

(2) $\overrightarrow{\mathbf{E G}}(Q)$ has a sink if and only if $[\check{Q}]$ is a vertex in $\overrightarrow{\mathbf{E G}}(Q)$ and in this case $[\check{Q}]$ is the unique sink.

Proof. [Q $]$ ] belongs to $\overrightarrow{\mathbf{E G}}(Q)$ by construction and it is a source in $\overrightarrow{\mathbf{E G}}(Q)$ since all the vertices in $\widehat{Q}$ are green. If $[R]$ is another source, then all the vertices in $R$ are green and therefore it follows from Proposition 2.10 that $R \simeq \widehat{Q}$, proving the first point. Now if $[R]$ is a sink in $\overrightarrow{\mathbf{E G}}(Q)$, then all its vertices are red and therefore, it follows from Proposition 2.10 that $R \simeq \breve{Q}$, proving the second point. Conversely, if $[\check{Q}]$ is in $\overrightarrow{\mathbf{E G}}(Q)$, then it is a sink since all its non-frozen vertices are red.

The following statement rephrases Corollary 2.12. 
Proposition 2.13. Let $Q$ be a cluster quiver. Then green $(Q) \neq \varnothing$ if and only if there is a sink in $\overrightarrow{\mathbf{E G}}(Q)$. In this case, there is a natural bijection between green $(Q)$ and the set of oriented paths in $\overrightarrow{\mathbf{E G}}(Q)$ from its unique source to its unique sink.

As it is explained in Section 6, $\overrightarrow{\mathbf{E G}}(Q)$ is isomorphic to the Hasse graph of various partially ordered sets. In particular, it has the following essential property:

Proposition 2.14. Let $Q$ be a cluster quiver. Then $\overrightarrow{\mathbf{E G}}(Q)$ has no oriented cycles.

2.5. Existence, finiteness and lengths. Let $Q$ be a cluster quiver. We recall that if $\mathbf{i}=\left(i_{1}, \ldots, i_{l}\right)$ is a green sequence for $Q$, then the integer $l$ is called the length of $\mathbf{i}$ and is denoted by $\ell(\mathbf{i})$. For any $l \geqslant 0$, we set

and

$$
\begin{aligned}
& \operatorname{green}_{l}(Q)=\{\mathbf{i} \in \operatorname{green}(Q) \mid \ell(\mathbf{i})=l\}, \\
& \operatorname{green}_{\leqslant l}(Q)=\{\mathbf{i} \in \operatorname{green}(Q) \mid \ell(\mathbf{i}) \leqslant l\}
\end{aligned}
$$

$$
\begin{gathered}
\ell_{\min }(Q)=\min \left\{l \geqslant 0 \mid \operatorname{green}_{l}(Q) \neq \varnothing\right\} \in \mathbb{Z}_{\geqslant 0}, \\
\ell_{\max }(Q)=\max \left\{l \geqslant 0 \mid \operatorname{green}_{l}(Q) \neq \varnothing\right\} \in \mathbb{Z}_{\geqslant 0} \sqcup\{\infty\},
\end{gathered}
$$

with the conventions that $\ell_{\min }(Q)=\ell_{\max }(Q)=0$ if green $(Q)$ is empty.

It is clear that if $Q$ and $Q^{\prime}$ are isomorphic quivers, then the isomorphism $\phi: Q \longrightarrow Q^{\prime}$ induces an isomorphism $\overrightarrow{\mathbf{E G}}(Q) \longrightarrow \overrightarrow{\mathbf{E G}}\left(Q^{\prime}\right)$ so that $\operatorname{green}_{l}(Q)=$ green $_{l}\left(Q^{\prime}\right)$ for any $l \geqslant 1$. The following proposition shows a similar result for oppositions:

Proposition 2.15. Let $Q$ be a cluster quiver. Then for any $l \geqslant 1$, there exists a natural bijection

$$
\operatorname{green}_{l}(Q) \leftrightarrow \operatorname{green}_{l}\left(Q^{\mathrm{op}}\right) .
$$

Proof. Let $\mathbf{i}=\left(i_{1}, \ldots, i_{l}\right)$ be a maximal green sequence. Then there exists $\pi \in \mathfrak{S}_{Q_{0}}$ such that $\mu_{\mathbf{i}}(\widehat{Q})=\pi \cdot \check{Q}$. Moreover, since $\pi$ fixes the frozen vertices and since the only arrows between frozen and non-frozen vertices in $\breve{Q}$ are the $i^{\prime} \longrightarrow i$ for $i \in Q_{0}$, the permutation $\pi$ is uniquely determined. Therefore we have $\mu_{\pi^{-1}\left(i_{1}\right)} \circ \cdots \circ \mu_{\pi^{-1}\left(i_{l}\right)}(\breve{Q})=\widehat{Q}$ where $\pi^{-1}\left(i_{l}\right)$ is red in $\breve{Q}$ and for any $2 \leqslant k \leqslant l$, the vertex $\pi^{-1}\left(i_{k}\right)$ is red in $\mu_{\pi^{-1}\left(i_{k+1}\right)} \circ \cdots \circ \mu_{\pi^{-1}\left(i_{l}\right)}(\check{Q})$. Since the mutations commute with taking opposite quivers, $\pi^{-1}\left(i_{l}\right)$ is green in $(\check{Q})^{\text {op }}$, the vertex $\pi^{-1}\left(i_{k}\right)$ is green in $\mu_{\pi^{-1}\left(i_{k+1}\right)} \circ \cdots \circ \mu_{\pi^{-1}\left(i_{l}\right)}\left((\breve{Q})^{\text {op }}\right)$ for any $2 \leqslant k \leqslant l$ and $\mu_{\pi^{-1}\left(i_{1}\right)} \circ$ $\cdots \circ \mu_{\pi^{-1}\left(i_{l}\right)}\left((\breve{Q})^{\mathrm{op}}\right)$ has only red vertices. Since $(\breve{Q})^{\mathrm{op}}=\widehat{Q^{\mathrm{op}}}$, it follows that $\left(\pi^{-1}\left(i_{l}\right), \ldots, \pi^{-1}\left(i_{1}\right)\right)$ is a maximal green sequence for $Q^{\mathrm{op}}$. We therefore get a map $\operatorname{green}_{l}(Q) \longrightarrow \operatorname{green}_{l}\left(Q^{\mathrm{op}}\right)$ and applying the same argument to $Q^{\mathrm{op}}$, we get its inverse. Therefore, it is a bijection.

Lemma 2.16. Let $Q$ be a cluster quiver and let $R, R^{\prime} \in \operatorname{Mut}(\hat{Q})$ such that $[R] \longrightarrow\left[R^{\prime}\right]$ in $\overrightarrow{\mathbf{E G}}(Q)$. Then $g\left(\left[R^{\prime}\right]\right) \geqslant g([R])-1$.

Proof. Assume that $R^{\prime}=\mu_{k}(R)$ for some green vertex $k$ in $R$. In order to prove the statement, it is enough to prove that any green vertex in $R$ which is different from $k$ is also green in $R^{\prime}$. We let $B=B(R)$ and $B^{\prime}=B\left(R^{\prime}\right)$ be the corresponding adjacency matrices. Let $i$ be a green vertex in $R$ and let $f$ be a frozen vertex in $R$. Since $i$ is green in $R$, we have $b_{i f} \geqslant 0$ and also, since $k$ is green in $R$, we have $b_{k f} \geqslant 0$. Therefore,

$$
\begin{aligned}
b_{i f}^{\prime} & =b_{i f}+\left[b_{i k}\right]_{+}\left[b_{k f}\right]_{+}-\left[b_{i k}\right]_{-}\left[b_{k f}\right]_{-} \\
& =b_{i f}+\left[b_{i k}\right]_{+}\left[b_{k f}\right]_{+} \\
& \geqslant b_{i f} \geqslant 0
\end{aligned}
$$

so that $i$ is green in $R^{\prime}$.

Remark 2.17. Note that under the hypothesis of Lemma 2.16, it may happen that $g\left(\left[R^{\prime}\right]\right)>g([R])-1$ since a red vertex in $R$ can turn green in $R^{\prime}$, see for instance the penultimate mutation in Figure 2. 
Corollary 2.18. Let $Q$ be a cluster quiver. If $\operatorname{green}(Q) \neq \varnothing$, then $\ell_{\min }(Q) \geqslant\left|Q_{0}\right|$.

Proof. By definition, in a maximal green sequence $\mathbf{i}=\left(i_{1}, \ldots, i_{l}\right)$, we have $g\left(\mu_{\mathbf{i}}(\hat{Q})\right)=0$ whereas $g(\widehat{Q})=$ $\left|Q_{0}\right|$. Therefore, it follows from Lemma 2.16 that $l \geqslant\left|Q_{0}\right|$.

Example 2.19. $\quad(1)$ Let $Q$ be the quiver $1 \longrightarrow 2 \longrightarrow 3$. Then $\mathbf{i}=(123)$ is a maximal green sequence and therefore $\ell_{\min }(Q)=3=\left|Q_{0}\right|$.

(2) Let $Q^{\prime}=\mu_{2}(Q)$ be the cyclic quiver with three vertices. Then it is easily verified that $\ell_{\min }\left(Q^{\prime}\right)=$ $4>3$ so that Corollary 2.18 only provides a lower bound for $\ell_{\min }$.

Note also that these examples show that $\ell_{\min }$ is not invariant under mutations. The same will appear to be true for $\ell_{\max }$.

We recall that for a quiver $Q$, an admissible numbering of $Q_{0}$ by sources (resp. by sinks) is an $n$-tuple $\left(i_{1}, \ldots, i_{n}\right)$ such that $Q_{0}=\left\{i_{1}, \ldots, i_{n}\right\}$ and where $i_{1}$ is a source (resp. a sink) in $Q$ and such that for any $2 \leqslant k \leqslant n$, the vertex $i_{k}$ is a source (resp. a sink) in $\mu_{i_{k-1}} \circ \cdots \circ \mu_{i_{1}}(Q)$.

Lemma 2.20. Let $Q$ be an acyclic quiver. Then any admissible numbering of $Q_{0}$ by sources is a maximal green sequence. In particular, green $(Q) \neq \varnothing$ and $\ell_{\min }(Q)=\left|Q_{0}\right|$.

Proof. Since $Q$ is acyclic, it is well-known that there is at least one admissible numbering of $Q_{0}$ by sources. Let $\mathbf{i}=\left(i_{1}, \ldots, i_{n}\right)$ be such a numbering. Without loss of generality, we assume that this admissible numbering is $(1, \ldots, n)$. For any $1 \leqslant k \leqslant n$, we let $B^{(k)}$ be the adjacency matrix of $R^{(k)}=\mu_{k} \circ \cdots \circ \mu_{1}(\widehat{Q})$ and $Q^{(k)}=\mu_{k} \circ \cdots \circ \mu_{1}(Q)$. We prove by induction on $k$ that the green vertices in $R^{(k)}$ are precisely $\{k+1, \ldots, n\}$.

Let $i \neq k$ be non-frozen vertices and $f$ be a frozen vertex. We have

$$
b_{i, f}^{(k)}=b_{i, f}^{(k-1)}+\left[b_{i, k}^{(k-1)}\right]_{+}\left[b_{k, f}^{(k-1)}\right]_{+}-\left[b_{i, k}^{(k-1)}\right]_{-}\left[b_{k, f}^{(k-1)}\right]_{-} .
$$

Since $k$ is a source in $Q^{(k-1)}$, it follows that $b_{i, k}^{(k-1)} \leqslant 0$. Also, by induction hypothesis $k$ is green in $R^{(k-1)}$ so that $b_{k, f}^{(k-1)} \geqslant 0$. Therefore, $b_{i, f}^{(k)}=b_{i, f}^{(k-1)}$ so that a non-frozen vertex $i \neq k$ is green (or red, respectively) in $R^{(k)}$ if and only if it is green (or red, respectively) in $R^{(k-1)}$. Moreover, $b_{k, k+n}^{(k)}=-b_{k, k+n}^{(k-1)}=-b_{k, k+n}=$ -1 so that $k$ is red in $R^{(k)}$ whereas it was green in $R^{(k-1)}$. Thus, the green vertices in $R^{(k)}$ are exactly $\{k+1, \ldots, n\}$. In particular, $(1, \ldots, n)$ is a maximal green sequence for $Q$.

In general, it is not true that $\operatorname{green}(Q) \neq \varnothing$ for an arbitrary quiver $Q$. For instance, we have the following proposition, which will be proved in Section 8 .

Proposition 2.21. The quiver

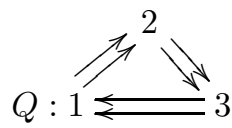

has no maximal green sequences.

More generally, a representation-theoretic criterion for the non-existence of maximal green sequences is given in Proposition 8.1. This in particular enables us to show that the McKay quiver

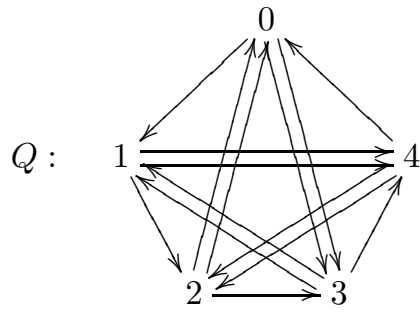


considered in 12 has no maximal green sequences either, see Example 8.2 .

The quiver in Proposition 2.21 is the quiver associated with any triangulation of the once-punctured torus, see [17. We will see in Section A.3 another example of a surface without boundary, namely the sphere with four punctures, for which there do exist maximal green sequences.

2.6. A conjecture on lengths. Based on the various examples we computed, we conjecture the following.

Conjecture 2.22. Let $Q$ be a cluster quiver. Then the set

$$
\left\{l \in \mathbb{Z}_{\geqslant 0} \mid \operatorname{green}_{l}(Q) \neq \varnothing\right\}
$$

is an interval in $\mathbb{Z}$.

Given a cluster quiver $Q$, the empirical maximal length is

$$
\ell_{\max }^{0}(Q)=\max \left\{l \geqslant 1 \mid \operatorname{green}_{k}(Q) \neq \varnothing \text { for any } k \text { s.t. } \ell_{\min }(Q) \leqslant k \leqslant l\right\}
$$

and we let

$$
\operatorname{green}^{0}(Q)=\operatorname{green}_{\leqslant \ell_{\max }^{0}(Q)}(Q),
$$

with the convention that $\ell_{\max }^{0}(Q)=0$ if $\operatorname{green}(Q)=\varnothing$.

With this terminology, Conjecture 2.22 states that $\ell_{\max }^{0}(Q)=\ell_{\max }(Q)$ and $\operatorname{green}^{0}(Q)=\operatorname{green}(Q)$. We will see several examples in which this appears.

The motivation for introducing the empirical maximal length is that it is easy to determine in practice: let $l$ be the smallest integer such that $\operatorname{green}_{l}(Q) \neq \varnothing$ and $\operatorname{green}_{l+1}(Q)=\varnothing$, then $l=\ell_{\max }^{0}(Q)$. Therefore, if Conjecture 2.22 holds, it is enough to find such an $l$ to determine green $(Q)$. This is the strategy we used in the computations whose results are given in Appendix $\mathrm{A}$

Note that Conjecture 2.22 does not hold true in the non-simply-laced case, as is shown for instance in Appendix A.1

\section{Maximal green Sequences and BPS Quivers}

As we already mentioned, maximal green sequences appear independently in theoretical physics, implicitly in 21] or more explicitly in [3]. In order to make the connection clear, we present in this short section a precise dictionary between the formal definition we gave in the previous section, and the physics review given in [11, §4.2].

We fix a cluster quiver $Q$. Vertices in $Q$ are called nodes in [11.

For simplicity, we identify the set $Q_{0}$ of vertices with $\{1, \ldots, n\}$. We let $\left\{\gamma_{i}\right\}_{1 \leqslant i \leqslant n}$ denote the canonical basis of $\mathbb{Z}^{n}$. In the terminology of [11, for any $R \in \operatorname{Mut}(\widehat{Q})$ and for any $1 \leqslant i \leqslant n$, the $i$ th $\mathbf{c}$-vector $\mathbf{c}_{i}(R) \in \mathbb{Z}^{n}$ is called the charge at node $i$. Therefore, the charges in $\hat{Q}$ are $\gamma_{1}, \ldots, \gamma_{n}$.

For any quiver $R \in \operatorname{Mut}(\widehat{Q})$ and for any $1 \leqslant k \leqslant n$, the charge at node $k$ in $R$ is $\mathbf{c}_{k}(R)=\sum_{i=1}^{n} c_{k ; i}(R) \gamma_{i}$ where $c_{k ; i}(R) \in \mathbb{Z}$ for any $i$. It follows from the sign-coherence for $\mathbf{c}$-vectors (see Theorem 2.6) that either $c_{k ; i}(R) \leqslant 0$ for every $i$, in which case $k$ is green in $R$, or $c_{k ; i}(R) \geqslant 0$ for every $i$, in which case $k$ is red in $R$. Moreover, if $k$ is green in $R$, then the c-vectors of $\mu_{k}(R)$ are precisely given in terms of those of $R$ by the rule for charges given in [11, (4.4)].

Now, the sequences of mutations considered in [11] for capturing complete BPS spectra are those for which:

(G1) the initial quiver appears with node charges $\gamma_{i}$;

(G2) the final quiver appears with node charges $-\gamma_{i}$;

(G3) At each step we may mutate on any node whose charge can be expressed as $\gamma=\sum_{i} c_{i} \gamma_{i}$ where $c_{i} \geqslant 0$ for any $1 \leqslant i \leqslant n$. 
Therefore, with our terminology, (G1) implies that the initial quiver $R$ has only green vertices, so that $[R]=[\widehat{Q}]$ according to Proposition 2.10 (G2) implies that the final quiver $R^{\prime}$ has only red vertices so that $[R]=[\check{Q}]$ according to Proposition [2.10. Finally, (G3) says that at each step in the mutation sequence $\widehat{Q} \simeq R \stackrel{\mu_{i_{1}}}{\longrightarrow} R^{(1)} \stackrel{\mu_{i_{l}}}{\longrightarrow} \cdots \stackrel{\mu_{i_{1}}}{\longrightarrow} R^{(l)} \simeq \check{Q}$, we mutated at a green vertex. Therefore, the sequences considered in [1] are precisely the maximal green sequences of $Q$.

\section{The Finite Cluster TYPE}

It was proved in [19] that a cluster algebra $\mathcal{A}_{Q}$ associated with a cluster quiver $Q$ has finitely many cluster variables if and only if $Q$ is mutation-equivalent to a Dynkin quiver $\vec{\Delta}$. In this case, $Q$ is called of finite cluster type and it is known that the number of cluster variables in $\mathcal{A}_{Q}$ equals the number of almost positive roots of the Dynkin quiver $\vec{\Delta}$, where the set $\Phi_{\geqslant-1}(\vec{\Delta})$ of almost positive roots of $\vec{\Delta}$ is the disjoint union of the set $\Phi_{+}(\vec{\Delta})$ of positive roots with the set of negative simple roots.

Theorem 4.1. Let $Q$ be a quiver of finite cluster type. Then

$$
\left|Q_{0}\right| \leqslant|\operatorname{green}(Q)|<\infty .
$$

Proof. Since $Q$ is of finite cluster type, the exchange graph $\mathbf{E G}(Q)$ is finite. Moreover, we know from Proposition 2.14 that $\overrightarrow{\mathbf{E G}}(Q)$ is acyclic. Hence, it contains only finitely many oriented paths and thus it follows from Proposition 2.13 that green $(Q)$ is finite.

Now since $\overrightarrow{\mathbf{E G}}(Q)$ is a finite acyclic oriented graph, it necessarily has at least one sink and one source and by Corollary 2.12 , it has a unique sink, corresponding to $[\hat{Q}]$, and a unique source, corresponding to $[\check{Q}]$. The underlying graph of $\overrightarrow{\mathbf{E G}}(Q)$ is $\left|Q_{0}\right|$-regular so that there are exactly $\left|Q_{0}\right|$ distinct arrows starting at $[\hat{Q}]$. Since $\overrightarrow{\mathbf{E G}}(Q)$ is finite, each of these arrows gives rise to at least one oriented path from the unique sink to the unique source and therefore we obtain at least $\left|Q_{0}\right|$ distinct oriented paths from the unique source to the unique sink in $\overrightarrow{\mathbf{E G}}(Q)$, that is, $\left|Q_{0}\right| \leqslant|\operatorname{green}(Q)|$.

Remark 4.2. (1) If $Q$ is a cluster quiver such that $\left|Q_{0}\right|=1$, then clearly $|\operatorname{green}(Q)|=1$.

(2) If $Q$ is a (connected) cluster quiver such that $\left|Q_{0}\right|=2$, then it is shown in Lemma 5.1 that green $(Q)$ has two elements of respective lengths 2 and 3 in the finite cluster type and a unique element, necessarily of length two, in the other cases.

(3) If $Q$ is a cluster quiver of finite cluster type such that $\left|Q_{0}\right|>2$, then it $|\operatorname{green}(Q)|>\left|Q_{0}\right|$ in general. In particular, one can observe that for linearly oriented quivers $Q_{n}$ of type $A_{n}$, the cardinality $\left|\operatorname{green}\left(Q_{n}\right)\right|$ seems to grow exponentially as a function of $n$, see [16].

Remark 4.3. If $Q$ is of finite cluster type, then a rough analysis provides an upper bound for $\ell_{\max }(Q)$. Namely, if we set

then we have

$$
\chi(Q)=|\{[R] \mid R \in \operatorname{Mut}(\hat{Q})\}|
$$

$$
\ell_{\max }(Q) \leqslant\left|Q_{0}\right| \cdot\left(\left|Q_{0}\right|-1\right)^{\chi(Q)-2} .
$$

Indeed, an oriented path on $\overrightarrow{\mathbf{E G}}(Q)$ starts at $[\hat{Q}]$ where we have $\left|Q_{0}\right|$ choices of directions and then, it passes at most once through any vertex in $\overrightarrow{\mathbf{E G}}(Q)$ distinct from $[\widehat{Q}]$ and $[\check{Q}]$. There are $\chi(Q)-2$ such vertices and at each such vertex $[R]$, there are at most $\left|Q_{0}\right|-1$ possible directions (since in order to leave $[R]$, we cannot use backwards the arrow we just used in order to arrive at $[R])$.

In general, these upper and lower bounds are not optimal but in the acyclic case, we can sharpen the result with the following theorem whose proof is given in Section 9 ,

Theorem 4.4. Let $Q$ be a Dynkin quiver. Then:

(1) $\ell_{\min }(Q)=\left|Q_{0}\right|$, 
(2) $\ell_{\max }(Q)=\left|\Phi_{+}(Q)\right|$, where $\Phi_{+}(Q)$ is the set of positive roots of $Q$.

Example 4.5. We show below the oriented exchange graphs for some quivers in the mutation class of type $A_{3}$. The labels on the faces correspond to denominators of the cluster variables in the corresponding clusters expressed in the seed with exchange matrix $B(Q)$. The unique source is circled in green and the unique sink is circled in red.

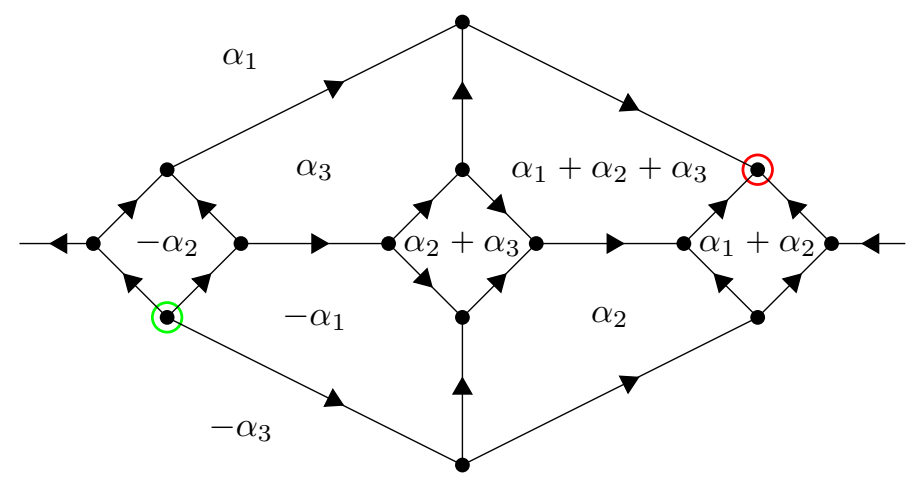

FiguRE 3 . The oriented exchange graph of $1 \longrightarrow 2 \longrightarrow 3$.

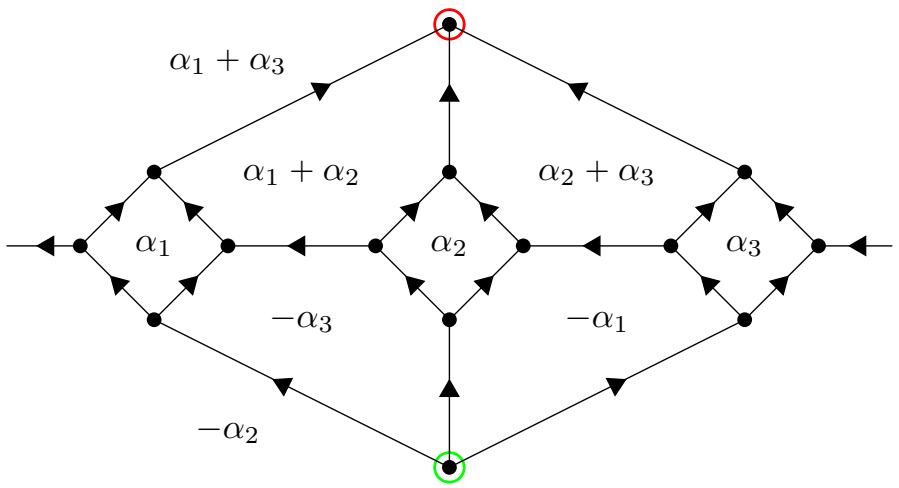

Figure 4. The oriented exchange graph of the cyclic quiver with 3 vertices.

We refer to Appendix $₫$ for additional examples.

\section{The INFinite Cluster TYPE}

If $Q$ is not of finite cluster type, then $\overrightarrow{\mathbf{E G}}(Q)$ is an infinite oriented graph and it is not known whether $\operatorname{green}(Q)$ is a finite set or not. Moreover, we have already seen in Proposition 2.21 that green $(Q)$ can be empty in the general case. When $Q$ is acyclic, we know from Corollary 2.18 that green $(Q)$ is non-empty so that we will now focus on this case.

It is proved in [19 that an acyclic quiver is of finite cluster type if and only if it is an orientation of a Dynkin diagram or, in representation-theoretic terms, if it is of finite representation type. Representationinfinite quivers are partitioned into two sets: affine quivers, which are acyclic orientations of extended 
Dynkin diagrams of types $\widetilde{A}, \widetilde{D}$ or $\widetilde{E}$, and wild quivers, which are the acyclic quivers which are neither Dynkin nor affine.

The following lemma will be proved in Section 10 .

Lemma 5.1. Let $Q$ be a (connected) cluster quiver with two vertices. Then:

(1) either $Q$ is of type $A_{2}$ and $\operatorname{green}(Q)=2, \ell_{\min }(Q)=2$ and $\ell_{\max }(Q)=3$,

(2) or $Q$ is representation-infinite and $\operatorname{green}(Q)=1$ and $\ell_{\min }(Q)=\ell_{\max }(Q)=2$.

5.1. The affine case. In the affine case, our main theorem is:

Theorem 5.2. Let $Q$ be an affine quiver. Then green $(Q)$ is finite and non-empty.

Example 5.3. Consider the quiver

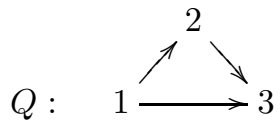

of affine type $\widetilde{A}_{2,1}$. Then locally around $[\hat{Q}]$, the oriented exchange graph $\overrightarrow{\mathbf{E G}}(Q)$ can be depicted as follows where the unique source is circled in green and the unique sink is circled in red. Here the faces are labelled by the denominator vectors of the cluster variables in the corresponding clusters, when expressed in the initial seed corresponding to $[\hat{Q}]$.

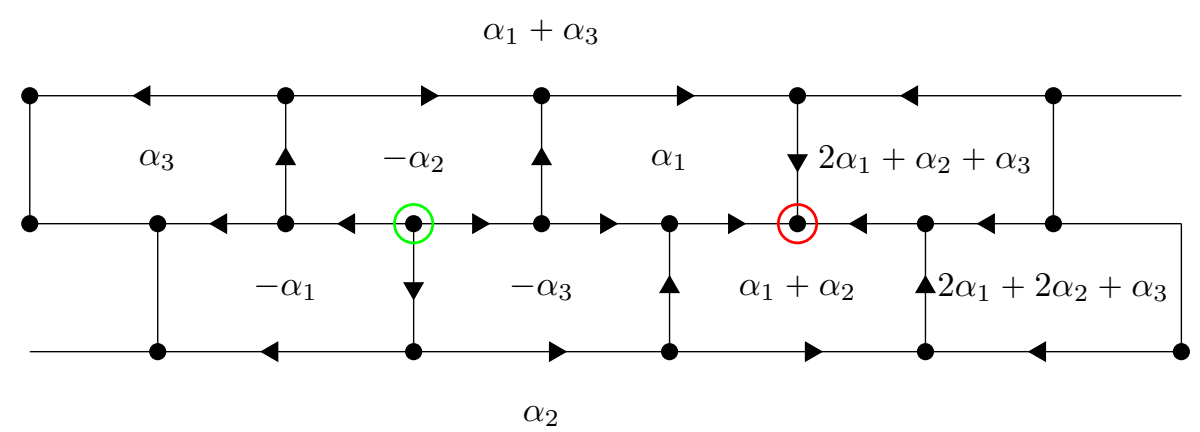

We see that, in this case, there are exactly five maximal green sequences.

For additional examples, we refer the reader to Appendix A.2

5.2. Wild quivers with three vertices. For the wild case, the situation appears to be more complicated. It is in fact known that for any (connected) wild quiver $Q$ with at least three vertices, there exist regular tilting $\mathrm{k} Q$-modules [44]. Therefore, the proof of Theorem 5.2 cannot be reproduced for wild quivers. However, we will prove in Proposition 10.2 that for quivers with three vertices, regular tilting $\mathbf{k} Q$-modules do not appear along maximal green sequences so that we are still able to deduce the finiteness of green $(Q)$ in this case.

Theorem 5.4. Let $Q$ be an acyclic quiver with three vertices. Then green $(Q)$ is finite and non-empty.

The proof is given in Section 10

If $Q$ is a wild quiver with at least four vertices, we do not know whether green $(Q)$ is finite or not. In this case, we could only compute a few examples which yield some evidence for the finiteness of this number.

As previously noticed, if Conjecture 2.22 holds, in order to prove that green $(Q)$ is a finite set for a given quiver $Q$, it is enough to find the smallest $l \geqslant 1$ such that $\operatorname{green}_{l}(Q) \neq \varnothing$ and such that $\operatorname{green}_{l+1}(Q)=\varnothing$; in this case green $(Q)=\operatorname{green}_{\leqslant l}(Q)$. We now provide an example of a wild quiver for which we computed such integers with the computer program Quiver Mutation Explorer [16]. 
Example 5.5. Consider the quiver

$$
Q: 1 \rightarrow 2 \rightarrow 3 \rightrightarrows 4
$$

Then

\begin{tabular}{c|c}
$l$ & $\left|\operatorname{green}_{l}(Q)\right|$ \\
\hline \hline 4 & 1 \\
\hline 5 & 7 \\
\hline 6 & 6 \\
\hline 7 & 7 \\
\hline 8 & 0
\end{tabular}

Therefore, $\ell_{\min }(Q)=4, \ell_{\max }^{0}(Q)=7$ and $\left|\operatorname{green}^{0}(Q)\right|=21$.

\section{Silting, tilting, Cluster-tilting And $t$-Structures}

As was already mentioned, given a cluster quiver $Q$, the oriented exchange graph $\overrightarrow{\mathbf{E G}}(Q)$ we are studying in this article is an orientation of the cluster exchange graph $\mathbf{E G}(Q)$ of the cluster algebra $\mathcal{A}_{Q}$, which is the dual graph of the cluster complex $\Delta\left(\mathcal{A}_{Q}\right)$ introduced in 19 . The same exchange graph also arises naturally in representation theory. This was first observed in [9, 10] where it was proved that if $Q$ is an acyclic quiver, then the clusters in $\mathcal{A}_{Q}$ correspond bijectively to the cluster-tilting objects in the so-called cluster category $\mathcal{C}_{Q}$ of $Q$ in such a way that cluster mutations correspond to mutations of cluster-tilting objects in $\mathcal{C}_{Q}$. This generalises to arbitrary skew-symmetric cluster algebras by considering the cluster-tilting theory of certain generalised cluster categories, see [4, 38. The aim of this section is to recall how $\mathbf{E G}(Q)$ and $\overrightarrow{\mathbf{E G}}(Q)$ arise in the context of additive categorifications and related topics in representation theory.

In the particular case where $Q$ is acyclic, identifying $\bmod \mathbf{k} Q$ with a subcategory of the cluster category $\mathcal{C}_{Q}$, the tilting $\mathbf{k} Q$-modules become cluster-tilting objects in $\mathcal{C}_{Q}$ and therefore, the cluster complex $\Delta\left(\mathcal{A}_{Q}\right)$ contains a certain subcomplex whose maximal simplices correspond to the tilting $\mathrm{k} Q$-modules. Already in 1987 Ringel observed that the set $\mathcal{T}_{A}$ of tilting modules over a finite dimensional algebra $A$ carries the structure of a simplicial complex. The study of this complex and of a poset structure on $\mathcal{T}_{A}$ was initiated in 42 and further carried out by Happel and Unger [47, 48, 23. We refer to the contributions of Ringel and of Unger in the Handbook of tilting theory for further details [45, 49].

In the first part of this section we recall the related notions for tilting modules, and then describe some generalisation to the setup of derived categories. We finally explain the link to cluster categories.

Throughout, we fix an algebraically closed field $\mathbf{k}$ and all the algebras we consider are $\mathbf{k}$-algebras. If there is no risk of confusion, for a finite-dimensional algebra $A$, we denote by $\mathcal{D}=\mathcal{D}^{b}(\bmod A)$ its bounded derived category with shift functor [1].

6.1. Tilting modules and their mutations. Let $A$ be a basic connected finite-dimensional k-algebra with $n$ non-isomorphic simple modules.

Definition 6.1 (Tilting modules). A finitely generated $A$-module $T$ is called tilting if

(1) $\operatorname{pdim} T \leqslant 1$,

(2) $\operatorname{Ext}_{A}^{i}(T, T)=0$ for all $i>0$,

(3) $A$ admits a coresolution in $\bmod A$ by $A$-modules in $\operatorname{add} T$.

A poset structure on the set $\mathcal{T}_{A}$ of isomorphism classes of basic tilting modules is defined in [42] by setting

$$
T \leqslant T^{\prime} \Leftrightarrow T^{\prime \perp} \subseteq T^{\perp}
$$

where

$$
T^{\perp}=\left\{X \in \bmod A \mid \operatorname{Ext}^{i}(T, X)=0 \text { for all } i>0\right\}
$$


We denote by $\overrightarrow{\mathcal{K}}_{\text {mod } A}$ the Hasse graph of this poset of tilting $A$-modules. If $A$ is hereditary, it is shown in 23 that the unoriented graph underlying this Hasse graph is the dual graph of the complex of tilting $A$ modules: there is an arrow $T \rightarrow T^{\prime}$ in $\overrightarrow{\mathcal{K}}_{\text {mod } A}$ precisely when $T=\oplus_{j} T_{j}$ and $T^{\prime}=\mu_{k}^{+}(T)=\left(T / T_{k}\right) \oplus T_{k}^{\prime}$ where $T_{k}^{\prime}$ is the forward mutation of $T$ at some $i$ defined as the cokernel of a minimal left add $\left(T / T_{k}\right)$ approximation $T_{k} \rightarrow M$ (we usually slightly abuse notations and write $T / T_{k}$ for $\oplus_{j \neq k} T_{j}$ ).

The poset $\mathcal{T}_{A}$ has $A$ as unique maximal element, and in case the algebra $A$ is Gorenstein, it has $D A$ as unique minimal element.

6.2. Silting objects and their mutations. The Hasse graph of the poset of tilting $A$-modules is not $n$-regular since not all tilting modules admit mutations. There are various ways to extend the notion of tilting module to a larger class of objects where mutations are always possible. We refer to [32] for a more complete picture on those various concepts, and we just recall the concept of silting objects here:

Let $\mathcal{D}$ denote the bounded derived category of $\bmod A$ with shift functor [1].

Definition 6.2 (Silting objects, 30]). An object $T$ in $\mathcal{D}$ is called silting if:

(1) $\operatorname{Hom}_{\mathcal{D}}(T, T[i])=0$ for any $i>0$,

(2) $\operatorname{thick}(T)=\mathcal{D}$

where thick $(T)$ denotes the thick subcategory generated by $T$ in $\mathcal{D}$.

It is shown in 2 that the set $\mathcal{T}_{\mathcal{D}}$ of isomorphism classes of basic silting objects is turned into a poset by setting

where as for modules

$$
T \leqslant T^{\prime} \Leftrightarrow T^{\prime \perp} \subseteq T^{\perp},
$$

$$
T^{\perp}=\left\{X \in \mathcal{D} \mid \operatorname{Hom}_{\mathcal{D}}(T, X[i])=0 \text { for all } i>0\right\} .
$$

Aihara and Iyama also show in 2 that the unoriented graph underlying the Hasse graph $\overrightarrow{\mathcal{K}}_{\mathcal{D}}$ of $\mathcal{T}_{\mathcal{D}}$ is the dual graph of the complex of silting objects in $\mathcal{D}$ : there is an arrow $T \rightarrow T^{\prime}$ in $\overrightarrow{\mathcal{K}}_{\mathcal{D}}$ precisely when $T=\oplus_{j} T_{j}$ and $T^{\prime}=\mu_{k}^{+}(T)=\left(T / T_{k}\right) \oplus T_{k}^{\prime}$ where $T_{k}^{\prime}$ is the forward mutation of $T$ at some $k$ defined as

$$
T_{k}^{\prime}=\text { Cone }\left(T_{k} \rightarrow \bigoplus_{j \neq k} \operatorname{Irr}\left(T_{k}, T_{j}\right)^{*} \otimes T_{j}\right) .
$$

A tilting object in $\mathcal{D}$ is a silting object $T$ such that $\operatorname{Hom}_{\mathcal{D}}(T, T[i])=0$ for any $i \neq 0$. In particular, any tilting $A$-module $T$ viewed as a stalk complex in $\mathcal{D}$ is a tilting object in $\mathcal{D}$, and therefore a silting object in $\mathcal{D}$. It follows immediately from the definition that if $T$ and $T^{\prime}$ are tilting $A$-modules such that $T^{\prime}=\mu_{k}^{+}(T)$ as $A$-modules, then $T^{\prime}=\mu_{k}^{+}(T)$ as silting objects in $\mathcal{D}$.

6.3. $t$-structures and their mutations. Let $T$ be a silting object in $\mathcal{D}$ and consider the full subcategories in $\mathcal{D}$ :

$$
\begin{gathered}
\mathcal{D}_{T}^{\leqslant 0}=\left\{N \in \mathcal{D} \mid \operatorname{Hom}_{\mathcal{D}}(T, N[i])=0 \text { for all } i>0\right\} \\
\mathcal{D}_{T}^{\geqslant 0}=\left\{N \in \mathcal{D} \mid \operatorname{Hom}_{\mathcal{D}}(T, N[i])=0 \text { for all } i<0\right\} .
\end{gathered}
$$

Then $\left(\mathcal{D}_{T}^{\leqslant 0}, \mathcal{D}_{T}^{\geqslant 0}\right)$ is a bounded $t$-structure on $\mathcal{D}$ with length heart $\mathcal{H}_{T}$ (that is, a heart in which every object has finite length), see for instance 32 . The simple forward mutation (also called forward tilt) of a heart of a bounded $t$-structure in $\mathcal{D}$ defined in 22. corresponds to the forward mutation of the respective silting object in $\mathcal{D}$, see [2, 32].

Also from these papers, we summarise the situation as follows: isomorphism classes of basic silting objects in $\mathcal{D}$ correspond bijectively to bounded $t$-structures with length heart in $\mathcal{D}$. The $t$-structures are ordered by inclusion of their left aisles, and the forward mutation describes the arrows in the Hasse graph of these posets, see Figure 5.

Since there is always an infinite number of silting objects in the derived category, we restrict our study to an interval with maximal element $A$ and minimal element $A[1]$, thus slightly larger than the poset of tilting $A$-modules. We denote by $\overrightarrow{\mathbf{E G}}_{\mathcal{D}}(A, A[1])$ the Hasse graph of the interval formed by the silting 

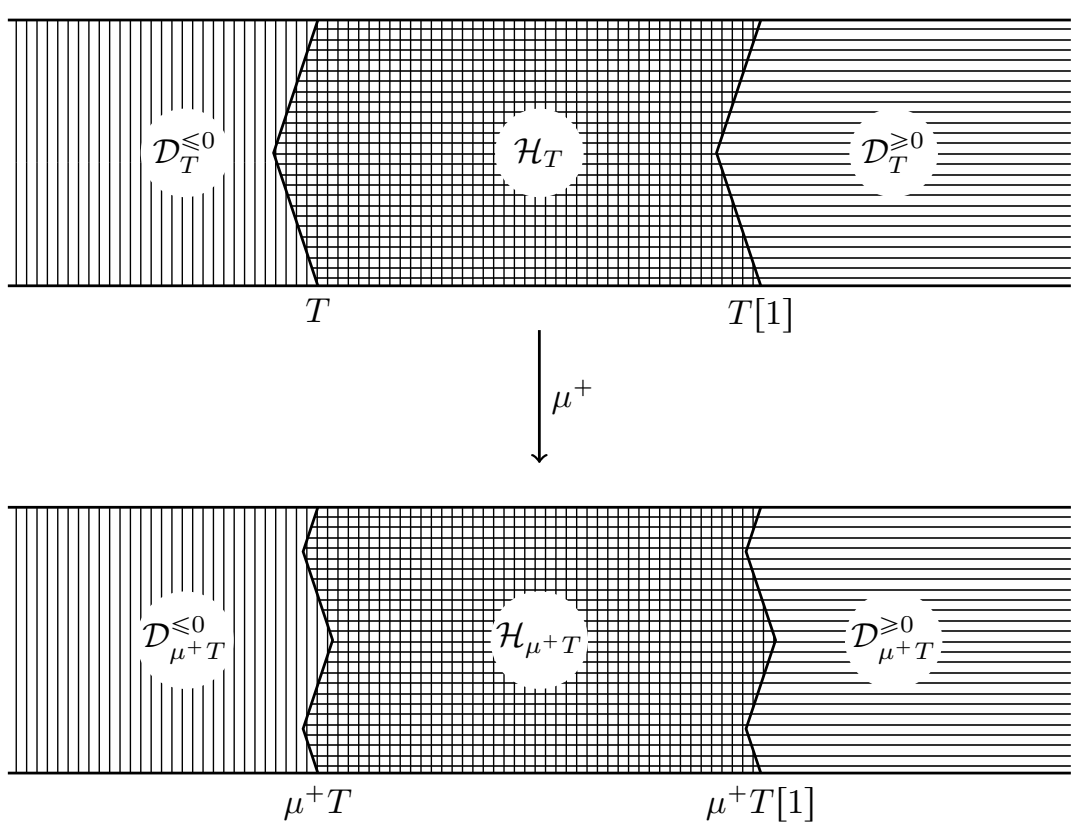

Figure 5. Forward mutation of a silting object in $\mathcal{D}$ and the inclusion of the corresponding left aisles.

objects which are between $A$ and $A[1]$ for this partial order. This interval, which was already considered in [31] appears to be relevant for the purpose of maximal green sequences, dilogarithm identities or for BPS quivers theory $[26,11,8]$.

\subsection{Cluster-tilting objects and their mutations.}

Definition 6.3 (Cluster-tilting objects). A cluster-tilting object $T$ in a triangulated category $\mathcal{C}$ is an object $T$ such that for any $X$ in $\mathcal{C}$, we have

$$
\operatorname{Ext}_{\mathcal{C}}^{1}(T, X)=0 \Leftrightarrow X \in \operatorname{add} T .
$$

Cluster-tilting objects were first considered in [9] where it was proved that the combinatorics of clustertilting objects in cluster categories were governed by mutations in (simply-laced) acyclic cluster algebras.

Given an acyclic quiver $Q$, its path algebra $\mathbf{k} Q$ is a finite-dimensional hereditary algebra. We denote by $\Gamma$ the Ginzburg dg-algebra associated with the quiver with potential $(Q, 0)$. It is a 3-Calabi-Yau dg-algebra concentrated in negative degrees, see 25]. We denote by $\mathcal{D} \Gamma$ the derived category of dg- $\Gamma$-modules, by per $\Gamma$ its perfect subcategory and by $\mathcal{D}_{\mathrm{fd}} \Gamma$ the full subcategory of $\mathcal{D} \Gamma$ formed by those dg-modules with finite-dimensional total homology. The cluster category of $Q$ is defined in [4] as the triangulated quotient $\mathcal{C}_{Q}=\operatorname{per} \Gamma / \mathcal{D}_{\mathrm{fd}} \Gamma$. It is a Hom-finite triangulated 2-CY category which is naturally triangle-equivalent to the cluster category defined as an orbit category in 9 .

Then $\mathbf{E G}(Q)=\overrightarrow{\mathbf{E G}}_{\mathcal{D} \mathbf{k} Q}(\mathbf{k} Q, \mathbf{k} Q[1])$ is an orientation of the graph of mutations of the cluster-tilting objects in $\mathcal{C}_{Q}$ and the unique source corresponds to the image of $\Gamma$ under the canonical morphism per $\Gamma \longrightarrow \mathcal{C}_{Q}$, see [28] and also [31, 32, 41].

For a general cluster quiver $Q$ and a non-degenerate potential $W$ on $Q$, it is still possible to form the triangulated quotient $\mathcal{C}_{Q, W}=$ per $\Gamma_{Q, W} / \mathcal{D}_{\mathrm{fd}} \Gamma_{Q, W}$ where $\Gamma_{Q, W}$ is the Ginzburg dg-algebra associated with the quiver with potential $(Q, W)$. Then $\overrightarrow{\mathbf{E G}}(Q)$ is an orientation of the connected component of 
the graph of mutations of cluster-tilting objects in $\mathcal{C}_{Q, W}$ which contains the image of $\Gamma_{Q, W}$ under the canonical morphism per $\Gamma_{Q, W} \longrightarrow \mathcal{C}_{Q, W}$.

If $\Sigma$ denotes the suspension functor in $\mathcal{D}_{\mathrm{fd}} \Gamma_{Q, W}$, a maximal green sequence for $Q$ corresponds in this context to a sequence of forward mutations from the canonical heart $\mathcal{H}$ of $\mathcal{D}_{\mathrm{fd}} \Gamma_{Q, W}$ to its shift $\Sigma \mathcal{H}$, see [26].

6.5. Patterns. Let $Q$ be a cluster quiver with $n$ vertices and $\mathbb{T}_{n}$ denote the $n$-regular tree so that the edges adjacent to any vertex in $\mathbb{T}_{n}$ are labelled by $\{1, \ldots, n\}$. Let $t_{0}$ be a vertex in that graph. To any vertex $t$ in $\mathbb{T}_{n}$ we can associate an ice quiver $Q(t)$ such that $Q\left(t_{0}\right)=\hat{Q}$ and such that $t$ and $t^{\prime}$ are joined by an edge labelled by $k$ in $\mathbb{T}_{n}$ if and only if $Q\left(t^{\prime}\right)=\mu_{k}(Q(t))$. This endows $\mathbb{T}_{n}$ with a structure of an oriented graph $\overrightarrow{\mathbb{T}}_{n}$ by orienting the edge $t \frac{k}{-} t^{\prime}$ towards $t^{\prime}$ if and only if $k$ is green in $Q(t)$.

Let $W$ be a non-degenerate potential on $Q$ and $\Gamma$ be the corresponding Ginzburg dg-algebra. The category $\mathcal{D}_{\mathrm{fd}} \Gamma$ (with suspension functor $\Sigma$ ) is endowed with a natural $t$-structure with length heart $\mathcal{H}$. As explained in [27, we can associate to any vertex $t$ in $\mathbb{T}_{n}$ a heart $\mathcal{H}(t)$ in $\mathcal{D}_{\mathrm{fd}} \Gamma$ such that $\mathcal{H}\left(t_{0}\right)=\mathcal{H}$ and such that there is an arrow $t \stackrel{k}{\longrightarrow} t^{\prime}$ in $\overrightarrow{\mathbb{T}}_{n}$ if and only if $\mathcal{H}\left(t^{\prime}\right)$ is obtained from $\mathcal{H}(t)$ by a forward mutation at the simple $S_{k}(t)$ in $\mathcal{H}(t)$, see also 31, 32. This is called the pattern of hearts in $\mathcal{D}_{\mathrm{fd}} \Gamma$. Then a maximal green sequence $\left(i_{1}, \ldots, i_{n}\right)$ corresponds to a path $t_{0} \stackrel{i_{1}}{\longrightarrow} t_{1} \stackrel{i_{2}}{\longrightarrow} \cdots \stackrel{i_{l}}{\longrightarrow} t_{l}$ in $\overrightarrow{\mathbb{T}}_{n}$ such that $\mathcal{H}\left(t_{l}\right) \simeq \Sigma \mathcal{H}(t)$.

If $Q$ is acyclic, $H=\mathbf{k} Q$ and $\mathcal{D}=\mathcal{D}^{b}(\bmod H)$ has shift functor [1], we can also associate to any vertex $t$ in $\mathbb{T}_{n}$ a silting object $T(t)$ in $\mathcal{D}$ such that $T\left(t_{0}\right)=\mathbf{k} Q$ and such that there is an arrow $t \stackrel{k}{\longrightarrow} t^{\prime}$ in $\vec{T}_{n}$ if and only if $T\left(t^{\prime}\right)$ is obtained from $T(t)$ by a forward mutation at $T_{k}^{(t)}$. This is called the silting pattern on $\mathcal{D}$. Then it follows from [31 that a maximal green sequence $\left(i_{1}, \ldots, i_{l}\right)$ corresponds to a path $t_{0} \stackrel{i_{1}}{\longrightarrow} t_{1} \stackrel{i_{2}}{\longrightarrow} \cdots \stackrel{i_{l}}{\longrightarrow} t_{l}$ in $\overrightarrow{\mathbb{T}}_{n}$ such that $T\left(t_{l}\right) \simeq H[1]$.

\section{More on the Happel-Unger Poset}

We assume in this section that $Q$ is a cluster quiver which admits a non-degenerate potential $W$ which is Jacobi-finite, that is, the Jacobian algebra $A=\mathcal{J}(Q, W)$ is finite-dimensional. These conditions are clearly satisfied when $Q$ is acyclic (with zero potential so that $\mathcal{J}(Q, 0)=\mathbf{k} Q$ ) or when $(Q, W)$ is given by an unpunctured surface, see 5,33 .

The Jacobian algebra $A$ is Gorenstein 29, thus we know from Section 6 that the oriented exchange graph $\overrightarrow{\mathcal{K}}_{\bmod A}$ of tilting modules has $A$ as unique maximal element and $D A$ as unique minimal element. We note that $\overrightarrow{\mathcal{K}}_{\text {mod } A}$ is in general not connected, even in cases where the exchange graph of silting objects is connected: Happel and Unger have shown that for an affine acyclic quiver $Q$, the $\operatorname{graph} \overrightarrow{\mathcal{K}}_{\bmod A}$ is connected precisely when $Q$ is not of type $\widetilde{A}_{1, s}$ with $s \geqslant 1$, or $\widetilde{A}_{2,2}$ with alternating orientation [23].

Theorem 7.1. Let $Q$ be an acyclic quiver and $H=\mathbf{k} Q$. Then $\overrightarrow{\mathcal{K}}_{\text {mod } H}$ is a full convex oriented subgraph of $\overrightarrow{\mathbf{E G}}(Q)$.

Proof. Let $T$ and $T^{\prime}$ be two tilting $H$-modules and consider a path

$$
T \longrightarrow T^{(1)} \longrightarrow \cdots \longrightarrow T^{(l)} \longrightarrow T^{\prime}
$$

in $\overrightarrow{\mathbf{E G}}(Q)$. Then we have the following chain of inclusions of the left aisles of the corresponding $t$ structures in $\mathcal{D}=\mathcal{D}(\bmod H)$ :

$$
\mathcal{D}_{T}^{\leqslant 0} \subset \mathcal{D}_{T^{(1)}}^{\leqslant 0} \subset \cdots \subset \mathcal{D}_{T^{(l)}}^{\leqslant 0} \subset \mathcal{D}_{T^{\prime}}^{\leqslant 0} .
$$

If there is some $1 \leqslant k \leqslant l$ such that $T^{(k)}$ is not a tilting $H$-module, then it is a silting object in $\overrightarrow{\mathbf{E G}}_{\mathcal{D}}(H, H[1])$ and therefore, it has a summand of the form $P_{i}[1]$. Thus we have $P_{i}[1] \in \mathcal{D}_{T^{(k)}}^{\leq 0}$ and we 
get $P_{i}[1] \in \mathcal{D}_{T^{\prime}}^{\leqslant 0}$ which implies

$$
0=\operatorname{Hom}_{\mathcal{D}}\left(P_{i}[1], T^{\prime}[1]\right)=\operatorname{Hom}_{\mathcal{D}}\left(P_{i}, T^{\prime}\right)=\operatorname{Hom}_{H}\left(P_{i}, T^{\prime}\right)
$$

so that $T^{\prime}$ is not sincere, which is a contradiction since every tilting $H$-module is sincere. Therefore, for any $1 \leqslant k \leqslant l$, the silting object $T^{(k)}$ is a tilting $H$-module. And as it was already mentioned, the forward mutation of a tilting module $T$ in $\bmod H$ coincides with the forward mutation of $T$ viewed as a silting object in $\mathcal{D}$. Therefore, $\overrightarrow{\mathcal{K}}_{\text {mod } H}$ is a full convex oriented subgraph of $\overrightarrow{\mathbf{E G}}(Q)$.

Example 7.2. Figure 6 shows how the Happel-Unger poset embeds in the oriented exchange graph of type $A_{2}$. In the $\mathrm{HU}$ poset, the unique source is circled in green and the unique sink is circled in red.

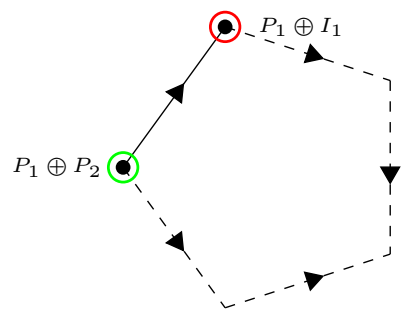

FIGURE 6 . The Happel-Unger poset, sitting in the oriented exchange graph of $1 \longrightarrow 2$.

Example 7.3. Figures 7 and 8 show how the Happel-Unger posets embed in certain oriented exchange graphs of type $A_{3}$. In both cases, the unique source of the $\mathrm{HU}$ poset is circled in green and the unique sink of the HU poset is circled in red.

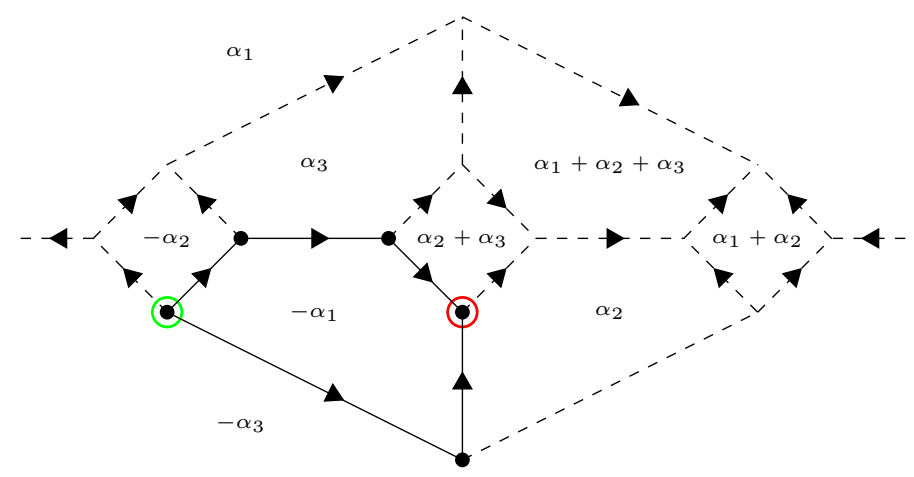

Figure 7 . The Happel-Unger poset for the quiver $1 \longrightarrow 2 \longrightarrow 3$, sitting in the poset of maximal green sequences, labelled with denominator vectors. 


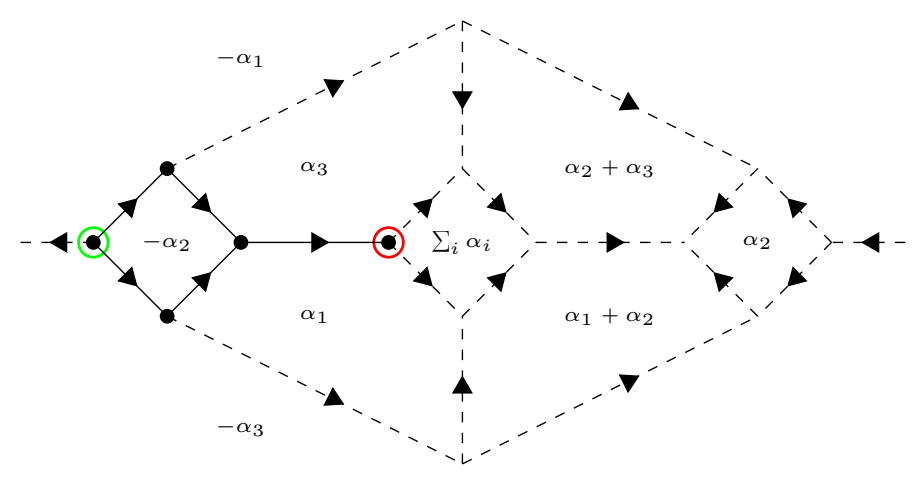

FIGURE 8. Happel-Unger poset, sitting in the oriented exchange graph of $1 \longleftarrow 2 \longrightarrow 3$, labelled with denominator vectors.

Remark 7.4. Example 7.3 shows a phenomenon which was already observed in [23, namely that the Happel-Unger poset is not stable under derived equivalences. It also shows that the oriented exchange graph is not stable under derived equivalences either. Indeed, in the linear case (Figure 7 ) there are 9 maximal green sequences whereas there are 10 in the alternating case (Figure 8).

Lemma 7.5. Let $Q$ be an acyclic quiver and $H=\mathbf{k} Q$. Let $\left(i_{1}, \ldots, i_{n}\right)$ be an admissible numbering of $Q_{0}$ by sinks. Assume that there is a path from $H$ to $D H$ in $\overrightarrow{\mathcal{K}}_{\bmod H}$ and let $\left(v_{1}, \ldots, v_{l}\right)$ be the corresponding green sequence for $Q$. Then $\left(v_{1}, \ldots, v_{l}, i_{1}, \ldots, i_{n}\right) \in \operatorname{green}_{l+n}(Q)$.

Proof. Since $D H$ is a tilting $H$-module, it is in particular a tilting object in $\mathcal{D}(\bmod H)$ and therefore a silting object in $\mathcal{D}=\mathcal{D}^{b}(\bmod H)$. Let $\left(i_{1}, \ldots, i_{n}\right)$ be an admissible numbering of $Q_{0}$ by sinks. The endomorphism algebra of $D H$ has Gabriel quiver $Q^{\text {op }}$ and $\left(i_{1}, \ldots, i_{n}\right)$ is an admissible sequence of sources for $Q^{\mathrm{op}}$. Therefore, considering successive APR-tilts at sources (see [7), we obtain a sequence of tilting objects in $\mathcal{D}$ :

where

$$
D H \stackrel{\mu_{i_{1}}}{\longrightarrow} T^{(1)} \stackrel{\mu_{i_{2}}}{\longrightarrow} \cdots \stackrel{\mu_{i_{n-1}}}{\longrightarrow} T^{(n-1)} \stackrel{\mu_{i_{n}}}{\longrightarrow} H[1]
$$

$$
T^{(k)}=\left(\bigoplus_{j<k} I_{i_{j}}\right) \oplus\left(\bigoplus_{l \leqslant k} \tau^{-1} I_{i_{j}}\right)=\left(\bigoplus_{j<k} I_{i_{l}}\right) \oplus\left(\bigoplus_{l \geqslant k} P_{i_{l}}[1]\right)
$$

for any $1 \leqslant k \leqslant n$. In particular, we have proper inclusions of left aisles

$$
\mathcal{D}_{D H}^{\leqslant 0} \subset \mathcal{D}_{T^{(1)}}^{\leqslant 0} \subset \mathcal{D}_{T^{(2)}}^{\leqslant 0} \subset \cdots \subset \mathcal{D}_{T^{(n-1)}}^{\leqslant 0} \subset \mathcal{D}_{H[1]}^{\leqslant 0}
$$

so that we obtain a path of length $n$ from $D H$ to $H[1]$ in $\mathbf{E G}(Q)$. By Theorem 7.1 a path of length $l$ from $H$ to $D H$ in $\overrightarrow{\mathcal{K}}_{\text {mod } H}$ gives rise to a path of length $l$ from $H$ to $D H$ in $\overrightarrow{\mathbf{E G}}(Q)$, composing this path with the above path from $D H$ to $H[1]$ gives a path from $H$ to $H[1]$ of length $n+l$ in $\overrightarrow{\mathbf{E G}}(Q)$, and therefore an element in $\operatorname{green}_{l+n}(Q)$. Figure 9 illustrates the proof.

The statement of Lemma 7.5 fails if $Q$ is not acyclic: in case $Q$ is the 3 -cycle with non-degenerate potential that 3 -cycle, the Jacobian algebra $A$ is self-injective and so the poset $\overrightarrow{\mathcal{K}}_{\text {mod } A}$ consists only of one point. The minimal length of a maximal green sequence is 4 , thus the statement of Lemma 7.5 does not hold.

Remark 7.6. Lemma 7.5 provides a criterion for the non-existence of paths from $H$ to $D H$ in $\overrightarrow{\mathcal{K}}_{\bmod H}$. For instance, consider the quiver

$$
Q: 1 \rightarrow 2 \rightarrow 3 \rightrightarrows 4 .
$$




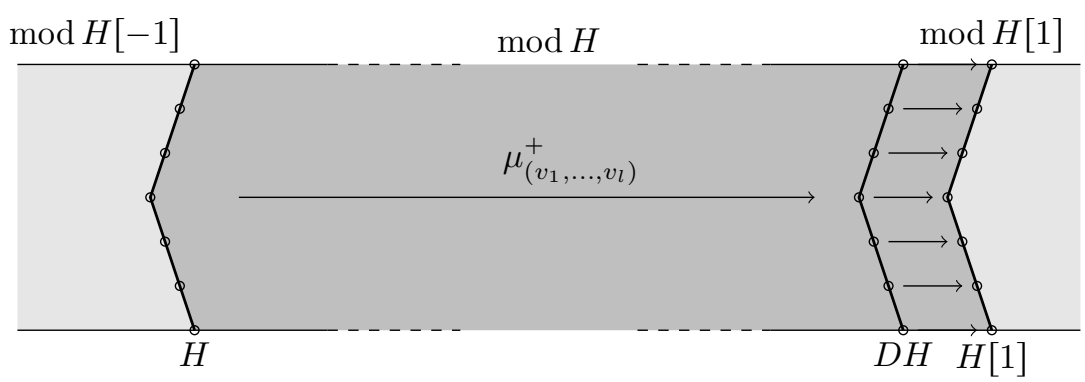

Figure 9. Extending a path from $H$ to $D H$ to a maximal green sequence.

Since $\mathbf{k} Q$ is wild hereditary, it has no projective-injective modules. Therefore, a path from $\mathbf{k} Q$ to $D \mathbf{k} Q$ in $\overrightarrow{\mathcal{K}}_{\text {mod } \mathbf{k} Q}$ must have a length at least 4 . However, as we saw in Example [5.5] we have $\ell_{\max }^{0}\left(Q_{1}\right)=7$. Therefore, if Conjecture 2.22 holds, then $\operatorname{green}_{l}(Q)$ is empty for $l \geqslant 8$ and there is no path from $\mathbf{k} Q$ to $D \mathbf{k} Q$ in $\overrightarrow{\mathcal{K}}_{\text {mod } \mathbf{k} Q}$.

\section{Proofs of Section 2}

\subsection{Proof of Proposition 2.10,}

Proof. Let $W$ be a generic potential on $Q$ and let $\Gamma$ be the Ginzburg dg-algebra associated with the quiver with potential $(Q, W)$. The category $\mathcal{D}_{\mathrm{fd}} \Gamma$ is endowed with a natural $t$-structure and we denote by $\mathcal{H}$ the corresponding heart with simples $S_{i}, i \in Q_{0}$. Let $\mathbb{T}_{\left|Q_{0}\right|}$ denote the $\left|Q_{0}\right|$-regular tree and consider the pattern of tilts $t \mapsto \mathcal{H}(t)$ with $\mathcal{H}\left(T_{0}\right)=\mathcal{H}$, see Section 6.5 or [27, §7.7].

Since $R \in \operatorname{Mut}(\hat{Q})$, there is some vertex $t$ in $\mathbb{T}_{\left|Q_{0}\right|}$ such that $R=Q(t)$ and since all the non-frozen vertices in $R$ are green, it means that all the simples in $\mathcal{H}(t)$ are in $\mathcal{H}$. Therefore, there exists a permutation $\pi \in \mathfrak{S}_{Q_{0}}$ such that $S_{i}(t) \simeq S_{\pi(i)}$ for any $i \in Q_{0}$ and it follows from [27, Corollary 7.11] that $\pi$ induces the wanted isomorphism of ice quivers.

Similarly, if all the non-frozen vertices in $R=Q(t)$ are red, it means that all the simples in $\mathcal{H}(t)$ are in $\Sigma \mathcal{H}$. Therefore, there exists a permutation $\pi \in \mathfrak{S}_{Q_{0}}$ such that $S_{i}(t) \simeq \Sigma S_{\pi(i)}$ for any $i \in Q_{0}$ and it follows from [27, Corollary 7.11] that $\pi$ induces the wanted isomorphism of ice quivers.

\subsection{Proof of Proposition 2.21,}

Proof. We know from Plamondon's thesis [39, Example 4.3] that there exists a (Jacobi-finite) nondegenerate potential $W$ on $Q$ such that there is no sequence of mutations in the cluster category $\mathcal{C}_{Q, W}$ joining the cluster-tilting object $\Gamma_{Q, W}$ to the cluster-tilting object $\Sigma \Gamma_{Q, W}$. Therefore, $\Gamma_{Q, W}$ and $\Sigma \Gamma_{Q, W}$ are in two different connected components of the mutation graph of cluster-tilting objects in $\mathcal{C}_{Q, W}$. In particular, if $\mathcal{H}$ denotes the canonical heart in $\mathcal{D}_{\mathrm{fd}} \Gamma_{Q, W}$, there is no sequence of forward mutations from $\mathcal{H}$ to $\Sigma \mathcal{H}$ and therefore, there is no path from $[\hat{Q}]$ to $[\breve{Q}]$ in $\overrightarrow{\mathbf{E G}}(Q)$. In other words, green $(Q)=\varnothing$.

8.3. On Jacobi-infinite quivers with potential. In this short section we give a criterion for the nonexistence of maximal green sequences. We recall that a quiver with potential is called Jacobi-infinite if the corresponding (completed) Jacobian algebra is infinite dimensional over $\mathbf{k}$.

Proposition 8.1. Let $(Q, W)$ be a Jacobi-infinite quiver with potential. Assume that it is non-degenerate. Then $\operatorname{green}(Q)=\varnothing$.

Proof. Let $\Gamma$ be the Ginzburg dg-algebra corresponding to $(Q, W)$. Let $\mathcal{H}$ denote the canonical heart in $\mathcal{D}_{\mathrm{fd}} \Gamma$. We claim that $\Sigma \mathcal{H}$ is not reachable from $\mathcal{H}$ by iterated forward mutations of hearts in $\mathcal{D}_{\mathrm{fd}} \Gamma$. 
Indeed, if so, we would obtain a sequence of mutations from $\Gamma$ to $\Sigma \Gamma$ in the generalised cluster category $\mathcal{C}$ associated with $(Q, W)$, see 27,38 . Therefore, $\Gamma$ and $\Sigma \Gamma$ are in the same connected component of the cluster-tilting graph of $\mathcal{C}$. Fix thus a sequence $\mathbf{i}=\left(i_{1}, \ldots, i_{l}\right)$ such that $\Gamma=\mu_{\mathbf{i}}(\Sigma \Gamma)$. Then it follows from [38, that this gives a sequence of mutations of decorated representation in the sense of [15] from the decorated representation of $(Q, W)$ corresponding to $\operatorname{Hom}_{\mathcal{C}}(\Gamma, \Sigma \Gamma)=0$ to the decorated representation of $\mu_{\mathbf{i}}(Q, W)$ of $\operatorname{Hom}_{\mathcal{C}}(\Gamma, \Gamma) \simeq \operatorname{End}_{\mathcal{C}}(\Gamma) \simeq \mathcal{J}(Q, W)$. However, $\mathcal{J}(Q, W)$ is infinite dimensional by hypothesis so that it is not a decorated representation in the sense of [15], a contradiction. Thus, there is no sequence of forward mutations from $\mathcal{H}$ to $\Sigma \mathcal{H}$ and therefore, there is no path from $[\widehat{Q}]$ to $[\breve{Q}]$ in $\overrightarrow{\mathbf{E G}}(Q)$. Hence, $\operatorname{green}(Q)=\varnothing$.

Example 8.2. Consider the McKay quiver

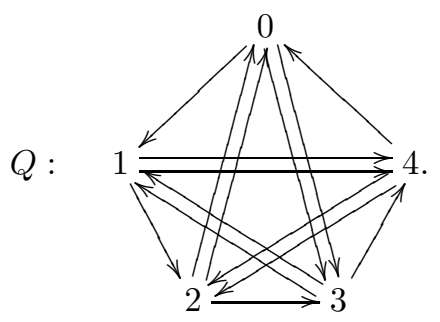

Then the main theorem of 12 asserts that $Q$ admits a non-degenerate potential such that the corresponding Jacobian algebra is infinite dimensional. Therefore, it follows from Proposition 8.1 that $Q$ has no maximal green sequences.

\section{Proofs of Section 4}

\subsection{Proof of Theorem 4.4.}

Proof. A Dynkin quiver is acyclic so that the first point follows from Lemma 2.20.

For the second point, consider the Weyl group $W$ associated with $Q$ with simple reflections $s_{i}$, with $i \in Q_{0}$. Let $w_{0}$ be the longest element in $W$ and fix a reduced expression $w_{0}=s_{i_{1}} \cdots s_{i_{r}}$ so that $r=\left|\Phi_{+}(Q)\right|$. Then it is well-known that one can choose $w_{0}$ in such a way that $\mathbf{i}=\left(i_{1}, \ldots, i_{r}\right)$ is an admissible sequence of $\operatorname{sinks}$ in $Q$. For any $1 \leqslant k \leqslant r$, we set

$$
T^{(k)}=\mu_{i_{k}}^{+} \circ \cdots \circ \mu_{i_{1}}^{+}(H)
$$

with the convention that $T^{(0)}=H$.

Since $\mathbf{i}$ is an admissible sequence of sinks, for any $1 \leqslant k \leqslant r$, the vertex $i_{k}$ is a sink in the quiver of the endomorphism ring of $T^{(k-1)}$ so that $T^{(k)}$ is obtained from $T^{(k-1)}$ by a simple APR-tilt (see [7). Therefore, the left aisles $\mathcal{D}_{T^{(k-1)}}^{\leqslant 0}$ and $\mathcal{D}_{T^{(k)}}^{\leqslant 0}$ differ by a single indecomposable object, namely $T_{i_{k}}^{(k-1)}$. Moreover, it is well-known that $T^{(r)} \simeq H[1]$. Therefore, we obtained a sequence of forward mutations

$$
H \stackrel{\mu_{i_{1}}^{+}}{\longrightarrow} T^{(1)} \stackrel{\mu_{i_{2}}^{+}}{\longrightarrow} \cdots \stackrel{\mu_{i_{r}}^{+}}{\longrightarrow} T^{(r)} \simeq H[1]
$$

which is the longest possible. Thus $\mathbf{i}$ is a maximal green sequence of the longest possible length and we have $\ell_{\max }(Q)=r=\left|\Phi_{+}(Q)\right|$.

Note that another proof of this result can be found in [40, Proposition 6.3]. 


\section{Proofs of Section [5]}

\subsection{Proof of Lemma 5.1 .}

Proof. The first point follows from Theorem 4.4. We now prove the second point. Let $\left(i_{1}, i_{2}\right)$ be an admissible numbering of $Q_{0}$ by sources. Then it was proved in Lemma 2.20 that $\left(i_{1}, i_{2}\right)$ is a maximal green sequence for $Q$. If there exists another maximal green sequence, then it is necessarily obtained by iterated mutations at sinks of the form $i_{2} i_{1} i_{2} i_{1} \cdots$. Let $H=\mathbf{k} Q, T^{(0)}=H$ and for any $k \geqslant 1$, let

$$
T^{(k)}= \begin{cases}\mu_{i_{2}}^{+}\left(T^{(k-1)}\right) & \text { if } k \text { is odd } \\ \mu_{i_{1}}^{+}\left(T^{(k-1)}\right) & \text { if } k \text { is even }\end{cases}
$$

Then, as in the proof of Theorem 4.4, the left aisles $\mathcal{D}_{T^{(k-1)}}^{\leqslant 0}$ and $\mathcal{D}_{T^{(k)}}^{\leqslant 0}$ differ by a single indecomposable object, namely $T_{i_{k}}^{(k-1)}$. However, the left aisle $\mathcal{D}_{H[1]}^{\leqslant 0}$ contains infinitely many more objects than the left aisle $\mathcal{D}_{H}^{\leqslant 0}$ so that $T^{(k)} \nsucceq H[1]$ for any $k \geqslant 1$. Therefore, there is no maximal green sequence beginning with $i_{2}$ and thus green $(Q)=\left\{\left(i_{1}, i_{2}\right)\right\}$.

10.2. Proof of Theorem $\mathbf{5 . 2}$, Let $Q$ be an affine quiver and let $H=\mathbf{k} Q$. The aim of this subsection is to prove that green $(Q)$ is a finite set. In fact, there are infinitely many green sequences starting at the silting object $H$, and we have to show that only finitely many of them can terminate at $H[1]$. To do that, we first recall some results from representation theory.

We let $S_{1}, \ldots, S_{n}$ denote the simple $H$-modules and for any $1 \leqslant i \leqslant n$, we denote by $P_{i}$ the projective cover of $S_{i}$ and by $I_{i}$ its injective hull.

As usual, we let $\mathcal{D}$ be the bounded derived category of $\bmod H$ with shift functor $[1]$. We denote by $\Gamma(\mathcal{D})$ the Auslander-Reiten quiver of $\mathcal{D}$, by $\mathcal{P}$ the connected component containing the projective $H$-modules and by $\mathcal{I}$ the connected component of $\Gamma(\mathcal{D})$ containing the injective $H$-modules. The indecomposable $H$-modules which are neither in $\mathcal{P}$ nor in $\mathcal{I}$ are referred to as regular modules.

We start with a general lemma:

Lemma 10.1. Let $H$ be a representation-infinite connected hereditary algebra. Then there exists $N \geqslant 0$ such that for any $k \geqslant N$, for any projective $H$-module $P$ and for any injective $H$-module $I$, the $H$-modules $\tau^{-k} P$ and $\tau^{k} I$ are sincere.

Proof. For any $1 \leqslant i \leqslant n$, it is known that the sets $\left\{\tau^{-k} P_{i}\right\}_{k \geqslant 0}$ and $\left\{\tau^{k} I_{i}\right\}_{k \geqslant 0}$ contain only finitely many non-sincere modules, see [6, Ch. IX, Proposition 5.6]. Therefore, there exists $N_{i} \geqslant 0$ such that $\tau^{-k} P_{i}$ and $\tau^{k} I_{i}$ are sincere for any $k \geqslant N_{i}$. Then $N=\max \left\{N_{i} \mid 1 \leqslant i \leqslant n\right\}$ is as wanted.

Proof of Theorem 5.2; Let $Q$ be an affine quiver and $H=\mathbf{k} Q$. Then a maximal green sequence for $Q$ is an oriented path

$$
H=T^{(0)} \longrightarrow T^{(1)} \longrightarrow \cdots \longrightarrow T^{(p-1)} \longrightarrow T^{(p)}=H[1]
$$

in $\overrightarrow{\mathcal{K}}_{\mathcal{D}}$ from $H$ to $H[1]$. Note that $H$ has its indecomposable summands in $\mathcal{P}$ and $H[1]$ has all of its indecomposable summands in $\mathcal{I}$.

Since the quiver $Q$ is affine there are only finitely many indecomposable rigid regular $H$-modules, and so the number of isomorphism classes of regular indecomposable summands of silting object arising on an oriented path from $H$ to $H[1]$ in $\overrightarrow{\mathcal{K}}_{\mathcal{D}}$ is finite. We want to show that the same holds for $\mathcal{P}$ and $\mathcal{I}$.

Let $N$ be as in Lemma 10.1, and suppose that one of the silting objects $T^{(s)}$ along the oriented path contains a summand of the form $\tau^{-k} P_{i}$ for an indecomposable projective $H$-module $P_{i}$ and $k \geqslant 2 N$. Every possible summand lying in $\mathcal{I}$ can be written as $\tau^{l} P_{j}[1]$ for some $l \geqslant 0$ and some indecomposable projective $H$-module $P_{j}$, and we have 


$$
\begin{aligned}
\operatorname{Ext}_{\mathcal{D}}^{1}\left(\tau^{l} P_{j}[1], \tau^{-k} P_{i}\right) & \simeq D \operatorname{Hom}_{\mathcal{D}}\left(\tau^{-k} P_{i}, \tau^{l+1} P_{j}[1]\right) \\
& \simeq D \operatorname{Hom}_{\mathcal{D}}\left(\tau^{-k-l} P_{i}, I_{j}\right) .
\end{aligned}
$$

But the space $\operatorname{Hom}_{\mathcal{D}}\left(\tau^{-k-l} P_{i}, I_{j}\right)$ is non-zero since $\tau^{-k-l} P_{i}$ is sincere by Lemma 10.1 and a sincere module maps nontrivially into every injective $I_{j}$. This shows that $T^{(s)}$ cannot contain both a summand of the form $\tau^{-k} P_{i}$ and a summand lying in $\mathcal{I}$. Moreover, it also follows from Lemma 10.1 that $\operatorname{Ext}_{\mathcal{D}}^{1}\left(\tau^{-m} P_{j}, \tau^{-k} P_{i}\right) \neq$ 0 for any $|m-k|>N$, thus all other possible summands of $T^{(s)}$ lying in $\mathcal{P}$ are of the form $\tau^{-m} P_{j}$ for some $N<m$ since we have chosen $k \geqslant 2 N$. By the same argument as above, such a summand would also extend with any object in $\mathcal{I}$.

Since $H$ is tame, any tilting object in $\mathcal{D}$ has at most $n-2$ indecomposable regular modules as direct summands, see [43. The same holds for any silting object $S$ such that $H \leqslant S \leqslant H[1]$. Thus, $T^{(s)}$ has at least two direct summands in $\mathcal{P}$, and therefore also $T^{(s+1)}$ cannot contain a summand in $\mathcal{I}$ since it still contains one of the two summands of $T^{(s)}$ lying in $\mathcal{P}$. This means the path would never terminate at $H[1]$, a contradiction. This proves that the number of isomorphism classes of indecomposable summands in $\mathcal{P}$ of a silting object arising on an oriented path from $H$ to $H[1]$ in $\overrightarrow{\mathcal{K}}_{\mathcal{D}}$ is finite. The proof of the same statement for $\mathcal{I}$ is dual.

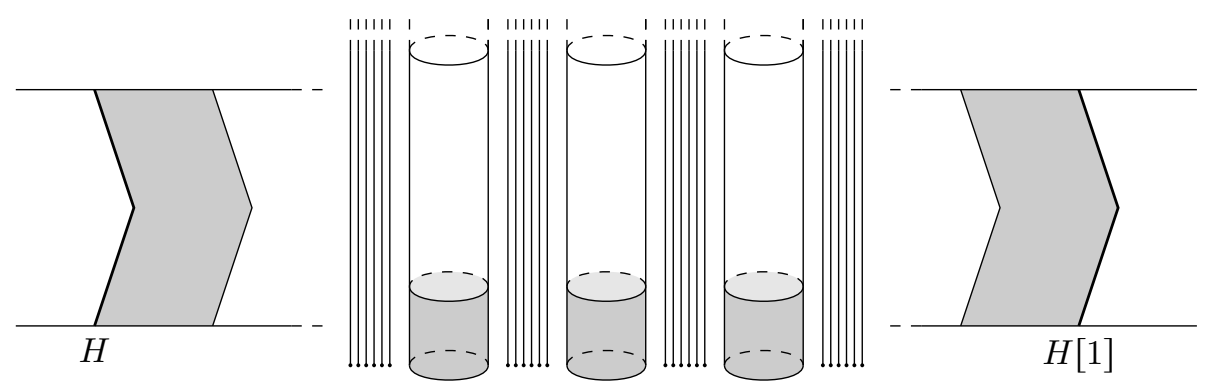

FiguRE 10. Schematic picture of the Auslander-Reiten quiver of $\mathcal{D}^{b}(\bmod H)$ for a tame hereditary algebra. Shaded areas correspond to where silting objects located on a path from $H$ to $H[1]$ can have their indecomposable summands.

10.3. Proof of Theorem 5.4. In this section, we want to prove that green $(Q)$ is a finite set for an acyclic quiver with 3 vertices.

We first show the following proposition.

Proposition 10.2. Let $Q$ be a connected wild quiver with three vertices and $H=\mathbf{k} Q$. Assume that $T$ is a silting object arising on a path from $H$ to $H[1]$ in $\overrightarrow{\mathbf{E G}}(Q)$. Then $T$ has at most one indecomposable regular summands.

Proof. We first prove that no regular $H$-module can appear along a maximal green sequence. Assume that $T^{\prime}$ is the first regular tilting $H$-module arising on a given maximal green sequence. Therefore, this sequence contains an arrow $T \stackrel{\mu_{v}}{\longrightarrow} T^{\prime}$ where $T^{\prime}=T / T_{v} \oplus T_{v}^{*}$ with $T_{v}$ preprojective, say $T_{v} \simeq \tau^{-s} P_{j}$, and $T_{v}^{*}, T / T_{v}$ regular. 
Since $T$ is tilting, we get

$$
\begin{aligned}
0 & =\operatorname{Ext}_{\mathcal{D}}^{1}\left(T / T_{v}, T_{v}\right) \\
& \simeq \operatorname{DHom}_{\mathcal{D}}\left(T_{v}, \tau\left(T / T_{v}\right)\right) \\
& \simeq \operatorname{DHom}_{\mathcal{D}}\left(\tau^{-s} P_{j}, \tau\left(T / T_{v}\right)\right) \\
& \simeq D \operatorname{Hom}_{\mathcal{D}}\left(P_{j}, \tau^{s+1}\left(T / T_{v}\right)\right) \\
& \simeq \operatorname{DHom}_{H}\left(P_{j}, \tau^{s+1}\left(T / T_{v}\right)\right) .
\end{aligned}
$$

Therefore, $\tau^{s+1}\left(T / T_{v}\right)$ is an $A /\left(e_{j}\right)$-module which is rigid (since $\tau^{s+1}\left(T / T_{v}\right)$ is rigid as an $A$-module) and, since it has $\left|Q_{0}\right|-1$ indecomposable summands, it is tilting as a $A /\left(e_{j}\right)$-module and thus, $\tau^{s+1} T^{\prime}=$ $\tau^{s+1}\left(T / T_{v}\right) \oplus \tau^{s+1} T_{v}^{*}$ is a regular tilting $H$-module satisfying the hypothesis of [47, Theorem 4.3]. Hence, any tilting module in the same connected component of $\overrightarrow{\mathcal{K}}_{\bmod H}$ as $\tau^{s+1} T^{\prime}$ contains at least two $\tau$-sincere indecomposable summands (that is, every module in their $\tau$-orbits is sincere). These indecomposables summands are in particular regular $H$-modules. In particular, any predecessor in $\overrightarrow{\mathcal{K}}_{\bmod H}$ of $\tau^{s+1} T^{\prime}$ has two regular summands and thefore, any predecessor in $\overrightarrow{\mathcal{K}}_{\bmod H}$ of $T^{\prime}$ has two regular summands, a contradiction. By convexity of $\overrightarrow{\mathcal{K}}_{\text {mod } H}$ inside $\overrightarrow{\mathbf{E G}}(Q)$ (Theorem [7.1), there is no path from $H$ to $T^{\prime}$ in $\overrightarrow{\mathbf{E G}}(Q)$.

Assume now that $T$ is a silting object on a maximal green sequence of the form $T=T / T_{v} \oplus T_{v}$ where $T / T_{v}$ is regular. By the previous discussion, $T_{v}$ cannot be regular. Assume that it is preprojective and set $T_{v}=\tau^{-s} P_{j}$ for some $s \geqslant 0$ and $j \in Q_{0}$ (the proof is dual for $T_{v}$ preinjective). If $v$ corresponds to a green vertex in $T$, there is an arrow $T \longrightarrow T^{\prime}=T / T_{v} \oplus T_{v}^{*}$ in $\overrightarrow{\mathbf{E G}}(Q)$. Now, as in the previous discussion, any predecessor in $\overrightarrow{\mathcal{K}}_{\text {mod } H}$ of $\tau^{s+1} T^{\prime}$ has two regular summands so that any predecessor in $\overrightarrow{\mathcal{K}}_{\text {mod } H}$ of $T^{\prime}$, and therefore of $T$, has two regular summands, a contradiction. Dually, if $v$ corresponds to a red vertex in $T$, there is an arrow $T^{\prime} \longrightarrow T$ in $\overrightarrow{\mathbf{E G}}(Q)$. As in the previous discussion, any successor in $\overrightarrow{\mathcal{K}}_{\bmod } H$ of $\tau^{s+1} T^{\prime}$ has two regular summands so that any successor in $\overrightarrow{\mathcal{K}}_{\bmod H}$ of $T^{\prime}$, and therefore of $T$, has two regular summands, a contradiction. By convexity of $\overrightarrow{\mathcal{K}}_{\bmod H}$ inside $\overrightarrow{\mathbf{E G}}(Q)$, there is no path from $H$ to $T$.

Proof of Theorem 5.4; Without loss of generality we can restrict to the case where $Q$ is connected. If it is Dynkin or affine, the result is known, see Theorems 4.1 and 5.2. We can thus restrict to the case where $Q$ is wild. We let $H=\mathbf{k} Q$. The number of maximal green sequences for $Q$ equals the number of paths from $H$ to $H[1]$ in $\overrightarrow{\mathbf{E G}}(Q)$.

Consider such a path and let $T$ be the last silting object along this path which is without summand of the form $\tau^{l} I_{i}$, with $l \geqslant-1$ and $i \in Q_{0}$. According to Proposition 10.2, $T$ has at most one regular direct summand. Dually, the first silting object without preprojective summand contains at most one regular direct summand. Then it follows from Lemma 10.1 that the non-regular summands of silting objects between $H$ and $H[1]$ run over a finite set of isomorphism classes.

Thus, if we consider a maximal green sequence, at the step before a regular summand possibly appears, the other two summands are necessarily preprojective or preinjective according to Proposition 10.2. In particular, it follows from Lemma 10.1 that there are only finitely many possible configurations for those two summands, and therefore a finite number of possible isomorphism classes of regular summands. Therefore, only finitely many isomorphism classes of silting objects can appear along a maximal green sequence and so that the number of maximal green sequences is finite.

\section{ACKNOWLEDGEMENTS}

The authors would like to thank Bernhard Keller and Dong Yang for interesting discussions on the topic. They would also like to thank an anonymous referee for valuable comments and corrections. The 
first author acknowledges support from NSERC and Bishop's University. The second author acknowledges support from the ANR Géométrie Tropicale et Algèbres Amassées.

\section{ApPendix A. Examples}

A.1. Rank two oriented exchange graphs. Any connected valued quiver with two vertices is either of infinite type or of type $A_{2}, B_{2}, C_{2}$ or $G_{2}$. We list below the corresponding oriented exchange graphs. It is interesting to observe that in the non-simply-laced types, the lengths of maximal green sequences do not form an interval.

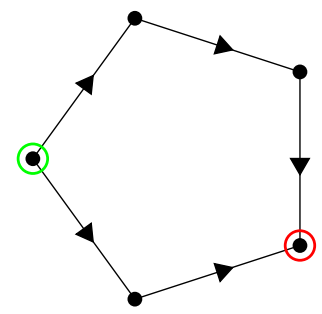

Figure 11. The oriented exchange graph of type $A_{2}$.

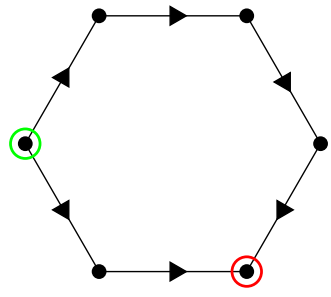

Figure 12. The oriented exchange graph of type $B_{2}$ or $C_{2}$.

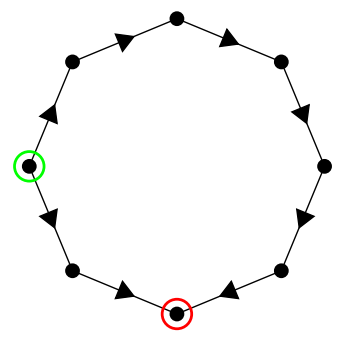

Figure 13. The oriented exchange graph of type $G_{2}$.

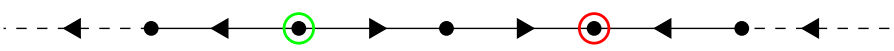

FiguRE 14. Oriented exchange graph of of rank two in infinite type. 
A.2. Examples of simply-laced affine types. For a quiver $Q$ of affine type, we have (non-maximal) green sequences of infinite lengths. However, Theorem 5.2 asserts that the number of maximal green sequences is finite. There is therefore a maximal length for the maximal green sequences. If Conjecture 2.22 holds, in order to list all the maximal green sequences, it is enough to find some $l \geqslant 1$ for which $0<\left|\operatorname{green}_{\leqslant l}(Q)\right|=\left|\operatorname{green}_{\leqslant l+1}(Q)\right|$ and then $\operatorname{green}(Q)=\operatorname{green}_{\leqslant l}(Q)$ and $\ell_{\max }^{0}(Q)=\ell_{\max }(Q)$. Using the Quiver Mutation Explorer, one can compute several such empirical lengths.

For $1 \leqslant n \leqslant 7$, if $Q$ is a quiver of type $\widetilde{A}_{n, 1}$, that is a quiver of type $\widetilde{A}$ with $n$ arrows going clockwise and one arrow going counterclockwise, we computed that for any $n \leqslant 7$, we have

$$
\ell_{\max }^{0}(Q)=\frac{n(n+3)}{2}
$$

For $4 \leqslant n \leqslant 7$, if $Q_{n}$ denotes the following quiver of type $\widetilde{D}_{n}$

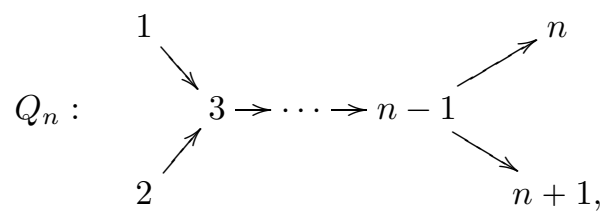

we obtained

$$
\ell_{\max }^{0}\left(Q_{n}\right)=2 n^{2}-2 n-2 .
$$

Also, it is interesting to note that for (acyclic) quivers of type $\widetilde{D}_{4}$, we could compute that the number of maximal green sequences depends on the choice of the orientation of the quiver but the minimal length and the empirical maximal length do not. We do not know if this is a general phenomenon.

A.3. An example from a surface without boundary. Consider the following triangulation $T$ of the sphere with four punctures.

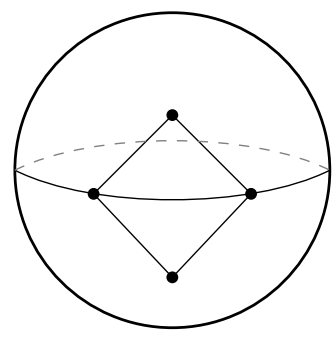

As defined in [17, the quiver $Q_{T}$ corresponding to this triangulation is the following.

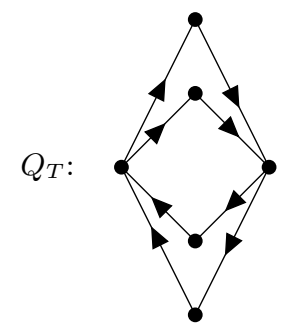

Then a direct computation shows that

$$
\ell_{\min }\left(Q_{T}\right)=12, \ell_{\max }^{0}\left(Q_{T}\right)=46
$$

and

$$
\mid \text { green }^{0}\left(Q_{T}\right) \mid=1044863666576 \text {. }
$$


A.4. An exceptional mutation-finite type. Consider the following quiver of type $\mathbb{X}_{6}$

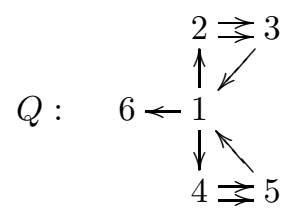

which first appeared in [14] as an example of mutation-finite quiver which is not arising from a surface. Then we obtained

$$
\ell_{\min }(Q)=10, \ell_{\max }^{0}(Q)=30 \text { and }\left|\operatorname{green}^{0}(Q)\right|=119819022 .
$$

We could not find a maximal green sequence for the type $\mathbb{X}_{7}$ appearing in [14].

\section{REFERENCES}

[1] T. Adachi, O. Iyama, and I. Reiten. $\tau$-tilting theory. arXiv:1210.1036v2 [math.RT], 2012.

[2] T. Aihara and O. Iyama. Silting mutation in triangulated categories. arXiv:1009.3370v3 [math.RT], 2010.

[3] M. Alim, S. Cecotti, C. Cordova, S. Espahbodi, A. Rastogi, and C. Vafa. N=2 quantum field theories and their BPS quivers. arXiv:1112.3984v1 [hep-th], 2011.

[4] C. Amiot. Cluster categories for algebras of global dimension 2 and quivers with potential. Ann. Inst. Fourier (Grenoble), 59(6):2525-2590, 2009.

[5] I. Assem, T. Brüstle, G. Charbonneau-Jodoin, and P. Plamondon. Gentle algebras arising from surface triangulations. Algebra and Number Theory, 4(2):201-229, 2010

[6] I. Assem, D. Simson, and A. Skowroński. Elements of the Representation Theory of Associative Algebras, Volume 1: Techniques of Representation Theory, volume 65 of London Mathematical Society Student Texts. Cambridge University Press, 2005.

[7] M. Auslander, M. Platzeck, and I. Reiten. Coxeter functors without diagrams. Trans. Amer. Math. Soc., 250:1-46, 1979 .

[8] T. Brüstle and G. Dupont. Double covers and maximal green sequences for surfaces. In preparation, 2012.

[9] A. Buan, R. Marsh, M. Reineke, I. Reiten, and G. Todorov. Tilting theory and cluster combinatorics. Adv. Math., 204(2):572-618, 2006.

[10] P. Caldero, F. Chapoton, and R. Schiffler. Quivers with relations arising from clusters $\left(A_{n}\right.$ case). Transactions of the $A M S, 358: 1347-1354,2006$.

[11] S. Cecotti, C. Córdova, and C. Vafa. Braids, walls and mirrors. arXiv:1110.2115v1 [hep-th], 2011.

[12] L. de Thanhoffer de Völcsey and M. Van den Bergh. Some new examples of non-degenerate quiver potentials. arXiv:1005.0808v3 [math.CO], 2010.

[13] L. Demonet. Mutations of group species with potentials and their representations. applications to cluster algebras. arXiv:1003.5078v2 [math.RT], 2010.

[14] H. Derksen and T. Owen. New graphs of finite mutation type. Electron. J. Combin., 15(1):Research Paper 139, 15, 2008.

[15] H. Derksen, J. Weyman, and A. Zelevinsky. Quivers with potentials and their representations II: applications to cluster algebras. J. Amer. Math. Soc., 23(3):749-790, 2010.

[16] G. Dupont and M. Pérotin. Quiver Mutation Explorer. available at http://mp-bull.github.com/qme-ng/, 2012.

[17] S. Fomin, M. Shapiro, and D. Thurston. Cluster algebras and triangulated surfaces. I. Cluster complexes. Acta Math., 201(1):83-146, 2008.

[18] S. Fomin and A. Zelevinsky. Cluster algebras I: Foundations. J. Amer. Math. Soc., 15:497-529, 2002.

[19] S. Fomin and A. Zelevinsky. Cluster algebras II: Finite type classification. Inventiones Mathematicae, 154:63-121, 2003.

[20] S. Fomin and A. Zelevinsky. Cluster algebras IV: Coefficients. Compositio Mathematica, 143(1):112-164, 2007.

[21] D. Gaiotto, G. Moore, and A. Neitzke. Wall-crossing, Hitchin systems, and the WKB approximation. arXiv:0907.3987v2 [hep-th], 2009

[22] D. Happel, I. Reiten, and S. O. Smalø. Tilting in abelian categories and quasitilted algebras. Mem. Amer. Math. Soc., 120(575):viii+ 88, 1996.

[23] D. Happel and L. Unger. On the set of tilting objects in hereditary categories. In Representations of algebras and related topics, volume 45 of Fields Inst. Commun., pages 141-159. Amer. Math. Soc., Providence, RI, 2005.

[24] B. Keller. Quiver mutation in Java. available at http://www.math.jussieu.fr/ ${ }^{\text {keller/quivermutation/. }}$

[25] B. Keller. Deformed Calabi-Yau completions. J. Reine Angew. Math., 654:125-180, 2011. With an appendix by Michel Van den Bergh.

[26] B. Keller. On cluster theory and quantum dilogarithm identities. arXiv:1102.4148v3 [math.RT], 2011. 
[27] B. Keller. Cluster algebras and derived categories. arXiv:1202.4161v4 [math.RT], 2012.

[28] B. Keller and P. Nicolás. Weight structures and simple dg modules for positive dg algebras. arXiv:1009.5904v3 [math.RT], 2010.

[29] B. Keller and I. Reiten. Cluster-tilted algebras are Gorenstein and stably Calabi-Yau. Adv. Math., 211(1):123-151, 2007.

[30] B. Keller and D. Vossieck. Aisles in derived categories. Bull. Soc. Math. Belg. Sér. A, 40(2):239-253, 1988. Deuxième Contact Franco-Belge en Algèbre (Faulx-les-Tombes, 1987).

[31] A. King and Y. Qiu. Exchange graphs of acyclic Calabi-Yau categories. arXiv:1109.2924v2 [math.RT], 2012.

[32] S. Koenig and D. Yang. Silting objects, simple-minded collections, $t$-structures and co-t-structures for finite-dimensional algebras. arXiv:1203.565\%v2 [math.RT], 2012.

[33] D. Labardini-Fragoso. Quivers with potentials associated to triangulated surfaces. Proc. London Math. Soc., 98(3):787839, 2009.

[34] S. Ladkani. Universal derived equivalences of posets of cluster tilting objects. arXiv:0710.2860v1 [math.RT], 2007.

[35] K. Nagao. Donaldson-Thomas theory and cluster algebras. arXiv:1002.4884v2 [math.AG], 2011.

[36] A. Nájera Chávez. On the c-vectors of an acyclic cluster algebra. arXiv:1203.1415v1 [math.RT], 2012.

[37] T. Nakanishi and A. Zelevinsky. On tropical dualities in cluster algebras. Contemp. Math., 565:217-226, 2012.

[38] P.-G. Plamondon. Cluster algebras via cluster categories with infinite-dimensional morphism spaces. Compos. Math., 147(6):1921-1934, 2011.

[39] P.-G. Plamondon. Generic bases for cluster algebras from the cluster category. arXiv:1111.4431v2 [math.RT], 2011.

[40] Y. Qiu. Stability conditions and quantum dilogarithm identities for Dynkin quivers. arXiv:1111.1010v2 [math.AG], 2011.

[41] Y. Qiu. c-sortable words as green mutation sequences. arXiv:1205.0034v1 [math. CO], 2012.

[42] C. Riedtmann and A. Schofield. On a simplicial complex associated with tilting modules. Comment. Math. Helv., 66(1):70-78, 1991.

[43] C. Ringel. Tame algebras and integral quadratic forms. Lecture Notes in Mathematics, 1099:1-376, 1984.

[44] C. M. Ringel. The regular components of the Auslander-Reiten quiver of a tilted algebra. Chinese Ann. Math. Ser. B, 9(1):1-18, 1988. A Chinese summary appears in Chinese Ann. Math. Ser. A 9 (1988), no. 1, 102.

[45] C. M. Ringel. Appendix: Some remarks concerning tilting modules and tilted algebras. Origin. Relevance. Future. In Handbook of tilting theory, volume 332 of London Math. Soc. Lecture Note Ser., pages 413-472. Cambridge Univ. Press, Cambridge, 2007.

[46] D. Speyer and H. Thomas. Acyclic cluster algebras revisited. arXiv:1203.0277v1 [math.RT], 2012.

[47] L. Unger. The partial order of tilting modules for three-point-quiver algebras. In Representation theory of algebras (Cocoyoc, 1994), volume 18 of CMS Conf. Proc., pages 671-679. Amer. Math. Soc., Providence, RI, 1996.

[48] L. Unger. The simplicial complex of tilting modules over quiver algebras. Proc. London Math. Soc. (3), 73(1):27-46, 1996.

[49] L. Unger. Combinatorial aspects of the set of tilting modules. In Handbook of tilting theory, volume 332 of London Math. Soc. Lecture Note Ser., pages 259-277. Cambridge Univ. Press, Cambridge, 2007.

Université de Sherbrooke, Département de Mathématiques, 2500 Boul. de L'Université, Sherbrooke QC J1K 2R1, CANADA.

E-mail address: tbruestl@ubishops.ca

Bishop's University, Department of Mathematics, 2600 College Street, Sherbrooke QC J1M 1Z7, Canada. E-mail address: Thomas.Brustle@usherbrooke.ca

Université Denis Diderot Paris 7, Paris, France.

E-mail address: dupontg@math.jussieu.fr

Bull SAS, Bruyères-le-ChÂtel, France.

E-mail address: matthieu.perotin@bull.net 